\title{
Dynamical Analysis of a Class of Prey-Predator Model with Beddington-DeAngelis Functional Response, Stochastic Perturbation, and Impulsive Toxicant Input
}

\author{
Feifei Bian, ${ }^{1}$ Wencai Zhao, ${ }^{1,2}$ Yi Song, ${ }^{1}$ and Rong Yue ${ }^{1}$ \\ ${ }^{1}$ College of Mathematics and Systems Science, Shandong University of Science and Technology, Qingdao 266590, China \\ ${ }^{2}$ State Key Laboratory of Mining Disaster Prevention and Control Co-Founded by Shandong Province and \\ the Ministry of Science and Technology, Shandong University of Science and Technology, Qingdao 266590, China
}

Correspondence should be addressed to Wencai Zhao; zhaowencai@sdust.edu.cn

Received 1 July 2017; Revised 8 October 2017; Accepted 17 October 2017; Published 5 December 2017

Academic Editor: Dimitri Volchenkov

Copyright (C) 2017 Feifei Bian et al. This is an open access article distributed under the Creative Commons Attribution License, which permits unrestricted use, distribution, and reproduction in any medium, provided the original work is properly cited.

\begin{abstract}
A stochastic prey-predator system in a polluted environment with Beddington-DeAngelis functional response is proposed and analyzed. Firstly, for the system with white noise perturbation, by analyzing the limit system, the existence of boundary periodic solutions and positive periodic solutions is proved and the sufficient conditions for the existence of boundary periodic solutions and positive periodic solutions are derived. And then for the stochastic system, by introducing Markov regime switching, the sufficient conditions for extinction or persistence of such system are obtained. Furthermore, we proved that the system is ergodic and has a stationary distribution when the concentration of toxicant is a positive constant. Finally, two examples with numerical simulations are carried out in order to illustrate the theoretical results.
\end{abstract}

\section{Introduction and Model Formulation}

The Lotka-Volterra model [1-3] is a classical model in the study of biological mathematics, and the continuous LotkaVolterra model which is modeled by ordinary differential equations and delay differential equations is widely used to characterize the dynamics of biological systems [4-13]. The functional response functions are important in the population ecological models [14]. In general, functional responses fall into two categories: one depends only on the density of the prey, such as Holling I-III [15-17]; the other depends on the density of both the prey and the predator, such as Beddington-DeAngelis type $[18,19]$. Compared with the Holling II functional response, the Beddington-DeAngelis type functional response, $F=f_{12} x y /\left(b_{2}+y+W_{12} x\right)$, has an additional term $y$ in the denominator modeling mutual interference among predators. In other words, this type of functional response is affected by both predator and prey. Some biologists believe that if the predators compete with each other to obtain food, functional response should depend on the density of both the prey and the predator. Arditi et al. [20] and Jost et al. [21, 22] used the actual observation data to verify this point. In particular, having collected observation data from 19 predator-prey communities, Skalski and Gilliam [23] found that predator-dependent functional responses were in agreement with the observation data, and in many instances, the Beddington-DeAngelis type looked better than the others. The Beddington-DeAngelis functional response has been widely used in the modeling of ecosystems in which there is mutual interference among predators [24, 25]. In [19], DeAngelis et al. have extensively investigated the dynamical properties of the following prey-predator system:

$$
\begin{aligned}
& \dot{x}(t) \\
& \quad=x(t)\left(a_{1}-\frac{f_{12} y(t)}{b_{2}+y(t)+W_{12} x(t)}-d_{1}-g_{1} x(t)\right), \\
& \dot{y}(t)=y(t)\left(\frac{e_{12} f_{12} x(t)}{b_{2}+y(t)+W_{12} x(t)}-d_{2}-g_{2} y(t)\right),
\end{aligned}
$$

where $x(t)$ and $y(t)$ represent the density of the prey and the predator, respectively. $a_{1}$ is the intrinsic growth rate of the 
prey, $f_{12}, b_{2}$, and $W_{12}$ are the consumption rate, the saturation constant, and the saturation constant for an alternative prey, respectively. $e_{12}$ is the conversion rate of nutrients into the reproduction for the predator. The parameters $d_{i}$ and $g_{i}(i=$ $1,2)$ are the nonpredatory loss rate and the interspecific competition rate. We refer the reader to [19] for more details.

In many ecosystems, predators tend to be omnivorous, they have wide variety of food sources. For example, the giant panda is omnivorous animal, since it can eat both meat and plant such as bamboos. In the lake ecosystem, some fishes not only prey on aquatic invertebrates, but also feed on algae and other aquatic plants. Polis and Strong in [26] and McCann and Hastings in [27] studied omnivorous nature of animals in the food chain in 1996 and 1997, respectively. Based on the above literature, we established a kind of omnivorous model as follows:

$$
\begin{aligned}
& \dot{x}(t)=x(t)\left(r_{1}-b_{1} x(t)-\frac{\lambda y(t)}{a+m y(t)+n x(t)}\right), \\
& \dot{y}(t)=y(t)\left(r_{2}+\frac{\gamma x(t)}{a+m y(t)+n x(t)}-b_{2} y(t)\right),
\end{aligned}
$$

where $r_{2}$ represents the growth rate of $y$ due to omnivorous nature and $b_{i}(i=1,2)$ denote the density-dependent coefficient of the prey and the predator, respectively. $\lambda, a, m$, $n$, and $\gamma$ are the consumption rate, the saturation constant, the predator interference, the saturation constant for an alternative prey, and the conversion rate, respectively. All parameters are positive in system (2).

It is well known that the biological population is inevitably affected by environment perturbation while the stochastic population model is more in line with the actual situation. Recently, various models based on stochastic differential equations (SDEs) have extensively been paid the attention of the researchers (see, e.g., [28-37]). Parameter perturbation induced by white noise is an important and common form to describe the effect of stochasticity (see, e.g., [37-48]). In this paper, we consider the white noise perturbation for the intrinsic growth rates of the prey and predator; that is, $r_{1} \rightarrow r_{1}+\sigma_{1} \dot{B}_{1}(t)$ and $r_{2} \rightarrow r_{2}+$ $\sigma_{2} \dot{B}_{2}(t)$, where $B_{1}(t), B_{2}(t)$ are mutually independent Brownian motions and $\sigma_{1}, \sigma_{2}$ denote the intensities of the white noise. On the other hand, it can be seen from the recent literature that the environmental pollution has an important effect on the population systems [49-60]. In 1983, Hallam et al. [61, 62] studied the influence of environmental pollution on the population and established a relationship model between environmental toxins and population. Subsequently, Hallam et al. [63, 64] studied the persistence and extinction of population in polluted environment. The mathematical model established by Hallam et al. considered only the toxins in the organism to cause a decrease in the birth rate or an individual death, which is reasonable in the case of lower concentration of the toxicant in the environment. When pollution is serious, the emission of pollutants may directly lead to the death of the species; see [65-69]. The authors in [68] added the environmental toxic term directly to the model; this is reasonable in the heavily polluted environment. For example, in a lake ecosystem, the discharge of large amounts of industrial waste water may directly lead to the death of fish, aquatic invertebrates, and so on. Therefore, we assume that the emission of pollutants to the environment is impulsive and directly affects the survival of the species in such an environment, so we get the following system:

$$
\begin{aligned}
& \mathrm{d} x(t)=x(t) \\
& \cdot\left(r_{1}-b_{1} x(t)-\frac{\lambda y(t)}{a+m y(t)+n x(t)}-\beta_{1} c_{e}(t)\right) \mathrm{d} t \\
& \quad+\sigma_{1}(t) x(t) \mathrm{d} B_{1}(t), \\
& \mathrm{d} y(t)=y(t) \\
& \quad\left(r_{2}+\frac{\gamma x(t)}{a+m y(t)+n x(t)}-b_{2} y(t)-\beta_{2} c_{e}(t)\right) \mathrm{d} t \\
& \quad+\sigma_{2}(t) y(t) \mathrm{d} B_{2}(t), \\
& \frac{\mathrm{d} c_{e}(t)}{\mathrm{d} t}=-h c_{e}(t), \\
& \Delta x(t)=0, \\
& \Delta y(t)=0, \\
& \Delta c_{e}(t)=\mu,
\end{aligned}
$$

where $\sigma_{1}(t), \sigma_{2}(t)$ are positive, nonconstant, and continuous functions of period $\tau, c_{e}(t)$ stands for the concentration of the toxicant in the environment, $h$ denotes the loss rate of toxicant at time $t, \tau$ is the impulsive input period and $\mu$ is the impulsive input amount, and $\beta_{1}$ and $\beta_{2}$ represent the dose-response of the prey and predator to the environmental toxicant, respectively.

Furthermore, the prey-predator model may be perturbed by telegraph noise which is distinguished by factors such as rain falls and nutrition and can be represented by switching among two or more regimes of environment [40, 60, 70-80]. For example, population growth rates in different seasons are not the same. The intraspecific competition coefficient varies according to the changes in nutrition and food resources. Generally, the switching between different regimes is memoryless and the waiting time for the next switch is exponentially distributed [81, 82]. Therefore, it can be described by a continuous-time Markov chain $r(t)$ taking values in a finite state space $\mathbb{S}=\{1,2, \ldots, m\}$. Taking into account the influences of white noise and telegraph noise, we propose the following stochastic differential system with impulsive toxicant input:

$$
\begin{aligned}
\mathrm{d} x & (t)=x(t)\left(r_{1}(r(t))\right. \\
- & \frac{\lambda(r(t)) y(t)}{a(r(t))+m(r(t)) y(t)+n(r(t)) x(t)} \\
& \left.-\beta_{1}(r(t)) c_{e}(t)-b_{1}(r(t)) x(t)\right) \mathrm{d} t+\sigma_{1}(r(t)) \\
& \cdot x(t) \mathrm{d} B_{1}(t),
\end{aligned}
$$




$$
\begin{aligned}
& \mathrm{d} y(t)=y(t)\left(r_{2}(r(t))\right. \\
& +\frac{\gamma(r(t)) x(t)}{a(r(t))+m(r(t)) y(t)+n(r(t)) x(t)} \\
& \left.\quad-\beta_{2}(r(t)) c_{e}(t)-b_{2}(r(t)) y(t)\right) \mathrm{d} t+\sigma_{2}(r(t)) \\
& \cdot y(t) \mathrm{d} B_{2}(t), \\
& \frac{\mathrm{d} c_{e}(t)}{\mathrm{d} t}=-h c_{e}(t), \\
& \Delta x(t)=0, \\
& \Delta y(t)=0, \\
& \Delta c_{e}(t)=\mu,
\end{aligned}
$$$$
t=k \tau \text {. }
$$

For any $k \in \mathbb{S}, r_{i}(k), b_{i}(k), \beta_{i}(k), \sigma_{i}(k)(i=1,2), \lambda(k)$, $a(k), m(k), n(k)$, and $\gamma(k)$ are all positive constants. In model (4), the population is inevitably affected by severe stochastic interference such as drought; the parameter switches one state $r(t)=i$ into another state $r(t)=j$ and it will switch into the next regime until the next major environmental change.

The rest of this paper is organized as follows. In Section 2, we provide preliminaries which are used in the following sections. In Section 3, we show that system (3) admits a nontrivial positive $\tau$-periodic solution by constructing Lyapunov function. In Section 4, we explore the sufficient conditions for extinction and permanence in mean of system (4). Finally, some examples with numerical simulations have been given to illustrate our theoretical results.

\section{Preliminaries}

Throughout this paper, let $\left(\Omega, \mathscr{F}, \mathscr{F}_{t \geq 0}, \mathbb{P}\right)$ be a complete probability space with a filtration $\mathscr{F}_{t \geq 0}$ satisfying the usual conditions, $B_{i}(t)(i=1,2)$ is one-dimensional Brownian motion on this space, and $r(t)$ is a right-continuous Markov chain and independent of the Brownian motion $B_{i}(t)$. The state space of this Markov chain is $\mathbb{S}=\{1,2, \ldots, m\}$. Suppose that the generator matrix of $r(t)$ is $\Gamma=\left(q_{i j}\right)_{1 \leq i, j \leq m}$, where $q_{i j}$ stands for the transition rate from state $i$ to $j$ and satisfies the following conditions:

$$
\begin{aligned}
\mathbb{P} & (r(t+\Delta t)=j \mid r(t)=i) \\
& = \begin{cases}q_{i j} \Delta t+o(\Delta t), & \text { if } i \neq j, \\
1+q_{i j} \Delta t+o(\Delta t), & \text { if } i=j ;\end{cases}
\end{aligned}
$$

here, $q_{i j} \geq 0$ if $i \neq j$, while $q_{i i}=-\sum_{i \neq j} q_{i j}, i, j=1,2, \ldots, m$. As a standing hypothesis, we assume that the Markov chain $r(t)$ is irreducible, which means that system (4) can switch from one regime to another. Under this assumption, the
Markov chain has a unique stationary distribution $\pi=$ $\left(\pi_{1}, \pi_{2}, \ldots, \pi_{m}\right)$ which is the solution of the system of linear equations $\pi \Gamma=0$ subject to $\sum_{j=1}^{m} \pi_{j}=1$ and $\pi_{j}>0$ for all $j \in \mathbb{S}$. Hence, for any vector $\emptyset=(\omega(1), \ldots, \omega(m))^{T}$, we have that

$$
\lim _{t \rightarrow \infty} \frac{1}{t} \int_{0}^{t} \omega(r(s)) d s=\sum_{k \in \mathbb{S}} \pi_{k} \varpi(k) .
$$

Let us consider the following stochastic differential equation with Markov conversion.

$$
\begin{aligned}
\mathrm{d} x(t) & =f(x(t), r(t)) \mathrm{d} t+g(x(t), r(t)) \mathrm{d} B(t), \\
x(0) & =x_{0}, \\
r(0) & =r_{0},
\end{aligned}
$$

where $x(t)=\left(x_{1}(t), \ldots, x_{n}(t)\right)^{T} \in \mathbb{R}^{n}, f: \mathbb{R}^{n} \times \mathbb{S} \rightarrow \mathbb{R}^{n}$, $g: \mathbb{R}^{n} \times \mathbb{S} \rightarrow \mathbb{R}^{n \times d}$, and $B(t)$ is a $d$-dimensional Brownian motion defined on the underlying probability space. The $n \times n$ matrix

$$
G(x, k)=g(x, k) g^{T}(x, k)=\left(G_{i j}\right)_{n \times n}
$$

is called the diffusion matrix. Let $V: \mathbb{R}^{n} \times \mathbb{S} \rightarrow \mathbb{R}^{n}$ be twice continuously differentiable and $\mathscr{L} V(x, k)$ which is defined as follows be the diffusion operator about $V(x, k)$ :

$$
\begin{aligned}
\mathscr{L} V(x, k)= & \sum_{i=1}^{n} f_{i}(x, k) \frac{\partial V(x, k)}{\partial x_{i}} \\
& +\frac{1}{2} \sum_{i, j=1}^{n} G_{i j} \frac{\partial^{2} V(x, k)}{\partial x_{i} \partial x_{j}} \\
& +\sum_{i \neq k \in \mathbb{S}} q_{k i}(V(x, i)-V(x, k)) .
\end{aligned}
$$

Particularly, for one-dimensional stochastic system

$$
\begin{aligned}
\mathrm{d} x(t)= & x(t)[a(r(t))-b(r(t)) x(t)] \mathrm{d} t \\
& +\alpha(r(t)) x(t) \mathrm{d} B(t), \\
x(0)= & x_{0}, \\
r(0)= & r_{0} ;
\end{aligned}
$$

the following two lemmas can be given from referring to the articles $[72,77]$.

Lemma 1. System (10) has a unique continuous positive solution $x(t)$. When it exists, the solution is global and stochastically ultimately bounded.

Lemma 2. Suppose that $\chi=\sum_{k \in \mathbb{S}} \pi_{k}\left[a(k)-(1 / 2) \alpha^{2}(k)\right] \neq 0$; then

(i) system (10) is stochastic permanent if and only if $\chi>0$;

(ii) system (10) is extinct if and only if $\chi<0$;

(iii) when $\chi>0$, system (10) is ergodic and there exists a unique stationary distribution $v(\cdot, \cdot)$, such that

$$
\chi=\sum_{k \in \mathbb{S}} b(k) \int_{\mathbb{R}_{+}} x v(\mathrm{~d} x, k) .
$$


Next, we consider the following stochastic differential equation:

$$
\mathrm{d} y(t)=f(t, y(t)) \mathrm{d} t+g(t, y(t)) \mathrm{d} B(t) .
$$

Lemma 3 (see [78]). Suppose that the coefficients of (12) are $\tau$-periodic in $t$ and there exists a function $V(t, y) \in C^{2}$ which is $\tau$-periodic in $t$, and $V(t, y)$ satisfies the following conditions:

(i) $\inf _{|y|>R} V(t, y) \rightarrow \infty$ as $R \rightarrow \infty$.

(ii) $L V(t, y) \leq-1$ outside some compact set.

Then there exists a solution for (12) which is a $\tau$-periodic Markov process.

Furthermore, we introduce some results from [80, 83] in Lemmas 4 and 5, which will be used in next section.

Lemma 4 (see $[80]$ ). Let $X(t) \in C\left[\Omega \times[0,+\infty), \mathbb{R}_{+}\right]$. Then

(i) if there are two positive constants $T$ and $m_{0}$ such that

$$
\ln X(t) \leq m t-m_{0} \int_{0}^{t} X(s) \mathrm{d} s+\sum_{k=1}^{n} \omega_{k} B_{k}(t)
$$

holds for all $t \geq T$ and constants $\omega_{k}(k=1,2, \ldots, n)$, then

$$
\begin{aligned}
\limsup _{t \rightarrow+\infty} \frac{1}{t} \int_{0}^{t} X(s) \mathrm{d} s & \leq \frac{m}{m_{0}}, \text { a.s. } \quad \text { if } m>0, \\
\lim _{t \rightarrow+\infty} X(t) & =0 \text {, a.s. if } m<0,
\end{aligned}
$$
that

(ii) if there are three positive constants $T, m$, and $m_{0}$ such

$$
\ln X(t) \geq m t-m_{0} \int_{0}^{t} X(s) \mathrm{d} s+\sum_{k=1}^{n} \omega_{k} B_{k}(t)
$$

holds for any $t \geq T$, then

$$
\liminf _{t \rightarrow+\infty} \frac{1}{t} \int_{0}^{t} X(s) \mathrm{d} s \geq \frac{m}{m_{0}} \text { a.s. }
$$

Finally, we give some basic properties of the following subsystem of system (3),

$$
\begin{aligned}
& \frac{\mathrm{d} c_{e}(t)}{\mathrm{d} t}=-h c_{e}(t), \quad t \neq k \tau, \quad k \in Z, \\
& \Delta c_{e}(t)=\mu, \quad t=k \tau, \quad k \in Z .
\end{aligned}
$$

Lemma 5 (see [83]). System (17) has a unique $\tau$-periodic solution $c_{e}^{*}(t)$ which is globally asymptotically stable. Here $c_{e}^{*}(t)=\mu e^{-h(t-k \tau)} /\left(1-e^{-h \tau}\right), t \in[k \tau,(k+1) \tau), c_{\max }=$ $\mu /\left(1-e^{-h \tau}\right)$, and $c_{\min }=\mu e^{-h \tau} /\left(1-e^{-h \tau}\right)$.

For convenience and simplicity, define $\widehat{\alpha}=\min _{i \in \mathbb{S}} \alpha_{i}$, $\check{\alpha}=\max _{i \in \mathbb{S}} \alpha_{i}$, and $\langle\varphi\rangle_{\theta}=(1 / \theta) \int_{0}^{\theta} \varphi(s) \mathrm{d} s$, where $\varphi(t)$ is an integrable function on $[0,+\infty)$. If $f$ is a bounded function on $[0,+\infty)$, define $f^{u}=\sup _{t \in[0,+\infty)} f(t)$.

\section{Existence of Periodic Solutions of System (3)}

In this section, we devote our attention to the investigation of the existence of periodic solutions of system (3). From Lemma 5, we know that system (17) has a globally asymptotically stable periodic solution $c_{e}^{*}(t)$; therefore, the limit system of (3) is

$$
\begin{aligned}
& \mathrm{d} x(t)=x(t) \\
& \quad \cdot\left(r_{1}-b_{1} x(t)-\frac{\lambda y(t)}{a+m y(t)+n x(t)}-\beta_{1} c_{e}^{*}(t)\right) \mathrm{d} t \\
& \quad+\sigma_{1}(t) x(t) \mathrm{d} B_{1}(t), \\
& \mathrm{d} y(t)=y(t) \\
& \quad \cdot\left(r_{2}+\frac{\gamma x(t)}{a+m y(t)+n x(t)}-b_{2} y(t)-\beta_{2} c_{e}^{*}(t)\right) \mathrm{d} t \\
& \quad+\sigma_{2}(t) y(t) \mathrm{d} B_{2}(t), \\
& x(0)=x_{0}, \\
& y(0)=y_{0},
\end{aligned}
$$

where $\sigma_{1}(t), \sigma_{2}(t)$, and $c_{e}^{*}(t)$ are all positive and continuous functions of period $\tau$.

Now, we discuss the existence of periodic solutions of system (18).

Define

$$
h_{i}=\frac{1}{\tau} \int_{0}^{\tau}\left(r_{i}-\beta_{i} c_{e}^{*}(s)-\frac{1}{2} \sigma_{i}^{2}(s)\right) \mathrm{d} s, \quad i=1,2 .
$$

Then, we have the following theorem about periodic solutions of system (18).

Theorem 6. If $h_{1}<0$ and $h_{2}>0$, there exists a prey extinction periodic solution $\left(0, y^{*}(t)\right)$ of system (18).

Proof. From the first equation of system (18), it is easy to see

$$
\begin{aligned}
\mathrm{d} x(t) \leq & x(t)\left[r_{1}-b_{1} x(t)-\beta_{1} c_{e}^{*}(t)\right] \mathrm{d} t \\
& +\sigma_{1}(t) x(t) \mathrm{d} B_{1}(t) .
\end{aligned}
$$

Applying Itô's formula and then integrating from 0 to $t$, we obtain

$$
\begin{aligned}
\ln x(t)-\ln x(0) \leq & \int_{0}^{t}\left(r_{1}-\beta_{1} c_{e}^{*}(s)-\frac{1}{2} \sigma_{1}^{2}(s)\right) \mathrm{d} s \\
& -\int_{0}^{t} b_{1} x(s) \mathrm{d} s+M(t),
\end{aligned}
$$

where $M(t)=\int_{0}^{t} \sigma_{1}(s) \mathrm{d} B_{1}(s)$ is local martingale. From strong law of large numbers for martingales (see [84]), we have

$$
\lim _{t \rightarrow \infty} \frac{M(t)}{t}=0, \quad \text { a.s. }
$$


It then follows from (21) by dividing $t$ on both sides and letting $t \rightarrow \infty$ that

$$
\begin{aligned}
\limsup _{t \rightarrow \infty} \frac{\ln x(t)}{t} & \leq\left\langle r_{1}-\beta_{1} c_{e}^{*}(s)-\frac{1}{2} \sigma_{1}^{2}(s)\right\rangle_{\tau} \\
& =r_{1}-\frac{\mu \beta_{1}}{\tau h}-\frac{1}{2}\left\langle\sigma_{1}^{2}(s)\right\rangle_{\tau}=h_{1}<0 ;
\end{aligned}
$$

namely, $x(t)$ tends to zero exponentially almost surely.

Since $\lim _{t \rightarrow \infty} x(t)=0$, a.s., from the second equation of system (18), its limit system is

$$
\begin{aligned}
\mathrm{d} y(t)= & y(t)\left(r_{2}-\beta_{2} c_{e}^{*}(t)-b_{2} y(t)\right) \\
& +\sigma_{2}(t) y(t) \mathrm{d} B_{2}(t) .
\end{aligned}
$$

According to Theorem 4.2 in [85], when $r_{2}-\beta_{2} c_{e}^{*}(t)>0$ and

$$
\begin{aligned}
& \left\langle r_{2}-\beta_{2} c_{e}^{*}(s)-\frac{1}{2} \sigma_{2}^{2}(s)\right\rangle_{\tau}=r_{2}-\frac{\mu \beta_{2}}{\tau h}-\frac{1}{2}\left\langle\sigma_{2}^{2}(s)\right\rangle_{\tau} \\
& =h_{2}>0
\end{aligned}
$$

(24) has a unique positive $\tau$-periodic solution $y^{*}(t)$.

Overall, when $h_{1}<0$ and $h_{2}>0$, there exists a prey extinction periodic solution $\left(0, y^{*}(t)\right)$ of system (18).

The proof of this theorem is completed.

In order to investigate the existence of a nontrivial positive $\tau$-periodic solution for system (18), first of all, we assume following conditions hold.

$$
\begin{aligned}
& \left(H_{1}\right) h_{i}>0, i=1,2 . \\
& \left(H_{2}\right) \lambda_{1}=\left(r_{1}-\beta_{1} c_{\min }+r_{1}-\beta_{1} c_{\max }\right)^{2}-4\left(r_{1}-\right. \\
& \left.\beta_{1} c_{\max }\right) h_{1}>0 \text { and } \xi_{1}>\left(\gamma^{2} / 4 b_{1} h_{2}\right) \lambda_{1} . \\
& \left(H_{3}\right) \xi_{2}=a b_{2} h_{1}-\lambda\left(r_{2}+\gamma / n-\beta_{2} c_{\min }\right)>0 \text { and } \\
& \left(\xi_{2} / a b_{1}\right)\left(r_{1}-\beta_{1} c_{\max }\right)>\left(b_{2} \xi_{1} / \gamma^{2}\right)\left[-h_{2}+\left(r_{2}+\gamma / n-\right.\right. \\
& \left.\left.\beta_{2} c_{\min }\right)\right] .
\end{aligned}
$$

Theorem 7. Suppose that $\left(H_{1}\right),\left(H_{2}\right)$, and $\left(H_{3}\right)$ hold, then there exists a positive $\tau$-periodic solution for system (18).

Proof. Obviously, the coefficients of system (18) are continuous bounded positive periodic functions in $t$. Now, we show that conditions (i) and (ii) of Lemma 3 hold. Define a nonnegative $C^{2}$-function

$$
\begin{aligned}
V(t, x, y)= & x-\frac{r_{1}-\beta_{1} c_{\max }}{b_{1}} \ln x+\frac{r_{1}-\beta_{1} c_{\max }}{b_{1}} \omega_{1}(t) \\
& -\frac{\xi_{1}}{\gamma^{2}} \ln y+\frac{\xi_{1}}{\gamma^{2}} \omega_{2}(t)+q y \\
=: & V_{1}+V_{2}+V_{3},
\end{aligned}
$$

where $V_{1}=x-\left(\left(r_{1}-\beta_{1} c_{\max }\right) / b_{1}\right) \ln x+\left(\left(r_{1}-\beta_{1} c_{\max }\right) / b_{1}\right) \omega_{1}(t)$, $V_{2}=-\left(\xi_{1} / \gamma^{2}\right) \ln y+\left(\xi_{1} / \gamma^{2}\right) \omega_{2}(t), V_{3}=q y, q=b_{2}\left(\left(\left(r_{1}-\right.\right.\right.$ $\left.\left.\left.\beta_{1} c_{\max }\right) / b_{1}\right) h_{1}+\left(\xi_{1} / \gamma^{2}\right) h_{2}\right) /\left(r_{2}-\beta_{2} c_{\min }+\gamma / n\right)^{2}$, and $\omega_{i}(t)$ is a function defined on $[0, \infty)$ satisfying $\omega_{i}^{\prime}(t)=r_{i}-(1 / 2) \sigma_{i}^{2}(t)-$ $\beta_{i} c_{e}^{*}(t)-h_{i}$ and $\omega_{i}(0)=0(i=1,2)$. Obviously, $\omega_{i}(t)$ is a $\tau$-periodic function on $[0, \infty)$. Therefore, the function $V(t, x, y)$ is $\tau$-periodic in $t$ and satisfies

$$
\liminf _{k \rightarrow \infty,(x, y) \in \mathbb{R}_{+}^{2} \backslash U_{k}} V(t, x, y)=\infty,
$$

where $U_{k}=(1 / k, k) \times(1 / k, k)$. Therefore, condition (i) of Lemma 3 holds. Next, we will prove that condition (ii) of Lemma 3 also holds.

Applying Itô's formula, one has

$$
\begin{aligned}
L V_{1} & =x\left[r_{1}-b_{1} x-\frac{\lambda y}{a+m y+n x}-\beta_{1} c_{e}^{*}(t)\right] \\
& -\frac{r_{1}-\beta_{1} c_{\max }}{b_{1}}\left[r_{1}-b_{1} x-\frac{\lambda y}{a+m y+n x}-\beta_{1} c_{e}^{*}(t)\right. \\
& \left.-\frac{\sigma_{1}^{2}(t)}{2}\right]+\frac{r_{1}-\beta_{1} c_{\max }}{b_{1}} \omega_{1}^{\prime}(t) \leq-b_{1} x^{2} \\
+ & {\left[\left(r_{1}-\beta_{1} c_{\min }\right)+\left(r_{1}-\beta_{1} c_{\max }\right)\right] x+\frac{\lambda}{b_{1} a}\left(r_{1}\right.} \\
- & \left.\beta_{1} c_{\max }\right) y-\frac{r_{1}-\beta_{1} c_{\max }}{b_{1}} h_{1}, \\
L V_{2} & =-\frac{\xi_{1}}{\gamma^{2}}\left(r_{2}+\frac{\gamma x}{a+m y+n x}-b_{2} y-\beta_{2} c_{e}^{*}(t)\right. \\
& \left.\leq \frac{\sigma_{2}^{2}(t)}{2}\right)+\frac{\xi_{1}}{\gamma^{2}} \omega_{2}^{\prime}(t) \leq \frac{\xi_{1} b_{2}}{\gamma^{2}} y-\frac{\xi_{1}}{\gamma^{2}} h_{2}, \\
L V_{3} & =q y\left[r_{2}+\frac{\gamma x}{a+m y+n x}-b_{2} y-\beta_{2} c_{e}^{*}(t)\right] \\
& q\left(r_{2}+\frac{\gamma}{n}-\beta_{2} c_{\min }\right) y-q b_{2} y^{2} .
\end{aligned}
$$

Therefore,

$$
\begin{aligned}
L V & \leq-b_{1} x^{2}+\left[\left(r_{1}-\beta_{1} c_{\min }\right)+\left(r_{1}-\beta_{1} c_{\max }\right)\right] x \\
& -\frac{r_{1}-\beta_{1} c_{\max }}{b_{1}} h_{1}-q b_{2} y^{2}+\left[\frac{\lambda\left(r_{1}-\beta_{1} c_{\max }\right)}{b_{1} a}\right. \\
& \left.+q\left(r_{2}+\frac{\gamma}{n}-\beta_{2} c_{\text {min }}\right)+\frac{\xi_{1} b_{2}}{\gamma^{2}}\right] y-\frac{\xi_{1}}{\gamma^{2}} h_{2} .
\end{aligned}
$$

Define a bounded closed set

$$
\mathscr{D}=\left\{(x, y) \in \mathbb{R}_{+}^{2}: \varepsilon \leq x \leq \frac{1}{\varepsilon}, \varepsilon \leq y \leq \frac{1}{\varepsilon}\right\},
$$

where $0<\varepsilon<1$ is a sufficiently small number such that

$$
\begin{aligned}
& {\left[\left(r_{1}-\beta_{1} c_{\min }\right)+\left(r_{1}-\beta_{1} c_{\max }\right)\right] \varepsilon \leq \frac{1}{2} \frac{r_{1}-\beta_{1} c_{\max }}{b_{1}} h_{1}+\frac{1}{2} \frac{\xi_{1}}{\gamma^{2}} h_{2}} \\
& -\frac{1}{2} \\
& \quad \cdot \frac{\left[\left(\lambda / b_{1} a\right)\left(r_{1}-\beta_{1} c_{\max }\right)+\xi_{1} b_{2} / \gamma^{2}+q\left(r_{2}-\beta_{2} c_{\min }+\gamma / n\right)\right]^{2}}{4 q b_{2}},
\end{aligned}
$$




$$
\begin{aligned}
& {\left[\frac{\lambda\left(r_{1}-\beta_{1} c_{\max }\right)}{b_{1} a}+q\left(r_{2}+\frac{\gamma}{n}-\beta_{2} c_{\min }\right)+\frac{\xi_{1} b_{2}}{\gamma^{2}}\right] \varepsilon} \\
& <-\frac{1}{8 b_{1}}\left[\left(r_{1}-\beta_{1} c_{\min }+r_{1}-\beta_{1} c_{\max }\right)^{2}-4\left(r_{1}-\beta_{1} c_{\max }\right) h_{1}\right. \\
& \left.\quad-\frac{4 b_{1} h_{2}}{\gamma^{2}} \xi_{1}\right] \\
& -\frac{b_{1}}{2 \varepsilon^{2}}+K_{3} \leq-1 \\
& -\frac{b_{2} q}{2 \varepsilon^{2}}+K_{4} \leq-1
\end{aligned}
$$

and $K_{3}, K_{4}$ are quantities to be determined in the rest of the proof.

Denote

$$
\begin{aligned}
& \mathscr{D}_{\varepsilon}^{1}=\left\{(x, y) \in \mathbb{R}_{+}^{2}: 0<x<\varepsilon\right\}, \\
& \mathscr{D}_{\varepsilon}^{2}=\left\{(x, y) \in \mathbb{R}_{+}^{2}: 0<y<\varepsilon\right\}, \\
& \mathscr{D}_{\varepsilon}^{3}=\left\{(x, y) \in \mathbb{R}_{+}^{2}: x>\frac{1}{\varepsilon}\right\}, \\
& \mathscr{D}_{\varepsilon}^{4}=\left\{(x, y) \in \mathbb{R}_{+}^{2}: y>\frac{1}{\varepsilon}\right\} .
\end{aligned}
$$

$$
\begin{gathered}
\frac{\lambda}{b_{1} a}\left(r_{1}-\beta_{1} c_{\max }\right)+\frac{\xi_{1} b_{2}}{\gamma^{2}}+q\left(r_{2}-\beta_{2} c_{\min }+\frac{\gamma}{n}\right)-2 \sqrt{q} \sqrt{b_{2}} \sqrt{\frac{r_{1}-\beta_{1} c_{\max }}{b_{1}} h_{1}+\frac{\xi_{1}}{\gamma^{2}} h_{2}} \\
=\frac{\left(\xi_{1} b_{2} / \gamma^{2}\right)\left[-h_{2}+\left(r_{2}+\gamma / n-\beta_{2} c_{\min }\right)\right]-\left(\xi_{2} / a b_{1}\right)\left(r_{1}-\beta_{1} c_{\max }\right)}{r_{2}+\gamma / n-\beta_{2} c_{\min }}<0 ;
\end{gathered}
$$

that is to say, $K_{1}<0$.

Case 2. If $(x, y) \in \mathscr{D}_{\varepsilon}^{2}$, from (29) and (32), we can get

$$
\begin{aligned}
L V & \leq-b_{1}\left[x-\frac{\left(r_{1}-\beta_{1} c_{\min }\right)+\left(r_{1}-\beta_{1} c_{\max }\right)}{2 b_{1}}\right]^{2} \\
& +\frac{1}{4 b_{1}}\left\{\left[\left(r_{1}-\beta_{1} c_{\min }\right)+\left(r_{1}-\beta_{1} c_{\max }\right)\right]^{2}\right. \\
& \left.-4\left(r_{1}-\beta_{1} c_{\max }\right) h_{1}\right\}-\frac{\xi_{1}}{\gamma^{2}} h_{2}+\left[\frac{\lambda\left(r_{1}-\beta_{1} c_{\max }\right)}{b_{1} a}\right. \\
& \left.+q\left(r_{2}+\frac{\gamma}{n}-\beta_{2} c_{\min }\right)+\frac{\xi_{1} b_{2}}{\gamma^{2}}\right] \varepsilon \\
& \leq \frac{1}{4 b_{1}}\left\{\left[\left(r_{1}-\beta_{1} c_{\min }\right)+\left(r_{1}-\beta_{1} c_{\max }\right)\right]^{2}\right. \\
& \left.-4\left(r_{1}-\beta_{1} c_{\max }\right) h_{1}-\frac{4 b_{1} \xi_{1}}{\gamma^{2}} h_{2}\right\} \\
& +\left[\frac{\lambda\left(r_{1}-\beta_{1} c_{\max }\right)}{b_{1} a}+q\left(r_{2}+\frac{\gamma}{n}-\beta_{2} c_{\min }\right)\right. \\
& \left.+\frac{\xi_{1} b_{2}}{\gamma^{2}}\right] \varepsilon \leq K_{2},
\end{aligned}
$$

Note that $\mathbb{R}_{+}^{2} \backslash \mathscr{D}=\mathscr{D}_{\varepsilon}^{1} \cup \mathscr{D}_{\varepsilon}^{2} \cup \mathscr{D}_{\varepsilon}^{3} \cup \mathscr{D}_{\varepsilon}^{4}$. Now, we prove $L V(t, x, y) \leq-1,(x, y) \in \mathbb{R}_{+}^{2} \backslash \mathscr{D}$.

Case 1. If $(x, y) \in \mathscr{D}_{\varepsilon}^{1}$, from (29), it implies that

$$
\begin{aligned}
L V & \leq-q b_{2}(y \\
& \left.-\frac{\left(\lambda / b_{1} a\right)\left(r_{1}-\beta_{1} c_{\max }\right)+\xi_{1} b_{2} / \gamma^{2}+q\left(r_{2}-\beta_{2} c_{\min }+\gamma / n\right)}{2 q b_{2}}\right)^{2} \\
& +\frac{\left[\left(\lambda / b_{1} a\right)\left(r_{1}-\beta_{1} c_{\max }\right)+\xi_{1} b_{2} / \gamma^{2}+q\left(r_{2}-\beta_{2} c_{\min }+\gamma / n\right)\right]^{2}}{4 q b_{2}} \\
& -\frac{r_{1}-\beta_{1} c_{\max }}{b_{1}} h_{1}-\frac{\xi_{1}}{\gamma^{2}} h_{2}+\left[\left(r_{1}-\beta_{1} c_{\min }\right)+\left(r_{1}-\beta_{1} c_{\max }\right)\right] \varepsilon \\
& \leq K_{1},
\end{aligned}
$$

where $K_{1}=(1 / 2)\left\{\left[\left(\lambda / b_{1} a\right)\left(r_{1}-\beta_{1} c_{\max }\right)+\xi_{1} b_{2} / \gamma^{2}+q\left(r_{2}-\right.\right.\right.$ $\left.\left.\left.\beta_{2} c_{\min }+\gamma / n\right)\right]^{2} / 4 q b_{2}-\left(\left(r_{1}-\beta_{1} c_{\max }\right) / b_{1}\right) h_{1}-\left(\xi_{1} / \gamma^{2}\right) h_{2}\right\}<0$. In fact, from condition $\left(\mathrm{H}_{3}\right)$, one can get 


$$
\begin{aligned}
& +\left[\frac{\lambda\left(r_{1}-\beta_{1} c_{\max }\right)}{a b_{1}}+\frac{\xi_{1} b_{2}}{\gamma^{2}}\right. \\
& \left.\left.+q\left(r_{2}-\beta_{2} c_{\min }+\frac{\gamma}{n}\right)\right] y-\frac{\xi_{1}}{\gamma_{2}} h_{2}\right\} .
\end{aligned}
$$

By (33), we have $L V \leq-1$.

Case 4. If $(x, y) \in \mathscr{D}_{\varepsilon}^{4}$, then

$$
\begin{aligned}
L V & \leq-\frac{b_{2} q}{2} y^{2}-b_{1} x^{2} \\
& +\left[\left(r_{1}-\beta_{1} c_{\min }\right)+\left(r_{1}-\beta_{1} c_{\max }\right)\right] x-\frac{r_{1}-\beta_{1} c_{\max }}{b_{1}} h_{1} \\
& -\frac{b_{2} q}{2} y^{2} \\
& +\left[\frac{\lambda\left(r_{1}-\beta_{1} c_{\max }\right)}{a b_{1}}+\frac{\xi_{1} b_{2}}{\gamma^{2}}+q\left(r_{2}-\beta_{2} c_{\min }+\frac{\gamma}{n}\right)\right] \\
& \cdot y-\frac{\xi_{1}}{\gamma^{2}} h_{2} \leq-\frac{q b_{2}}{2 \varepsilon^{2}}+K_{4},
\end{aligned}
$$

where

$$
\begin{aligned}
K_{4} & =\sup _{(x, y) \in \mathbb{R}_{+}^{2}}\left\{-b_{1} x^{2}+\left[\left(r_{1}-\beta_{1} c_{\min }\right)\right.\right. \\
& \left.+\left(r_{1}-\beta_{1} c_{\max }\right)\right] x-\frac{r_{1}-\beta_{1} c_{\max }}{b_{1}} h_{1}-\frac{q b_{2}}{2} y^{2} \\
& +\left[\frac{\lambda\left(r_{1}-\beta_{1} c_{\max }\right)}{a b_{1}}+\frac{\xi_{1} b_{2}}{\gamma^{2}}\right. \\
& \left.\left.+q\left(r_{2}-\beta_{2} c_{\min }+\frac{\gamma}{n}\right)\right] y-\frac{\xi_{1}}{\gamma^{2}} h_{2}\right\} .
\end{aligned}
$$

By (34), we obtain $L V \leq-1$.

Thus,

$$
\begin{aligned}
L V & \leq \min \left\{K_{1}, K_{2},-1\right\}, \\
(x, y) & \in \mathbb{R}_{+}^{2} \backslash \mathscr{D} .
\end{aligned}
$$

Therefore, the proof of Theorem 7 is completed.

\section{Extinction and Persistence in Mean of System (4)}

In this section, we investigate the long-term dynamic behaviors of the prey-predator system (4) with white noise and telegraph noise in a polluted environment and then discuss the extinction and average persistence of prey and predator. According to Lemma 5, the periodic solution $c_{e}^{*}(t)$ of the toxicant input is globally asymptotically stable, so the limit system of (4) is

$$
\begin{aligned}
& \mathrm{d} x(t)=x(t)\left(r_{1}(r(t))-b_{1}(r(t)) x(t)\right. \\
& -\frac{\lambda(r(t)) y(t)}{a(r(t))+m(r(t)) y(t)+n(r(t)) x(t)} \\
& \left.\quad-\beta_{1}(r(t)) c_{e}^{*}(t)\right) \mathrm{d} t+\sigma_{1}(r(t)) x(t) \mathrm{d} B_{1}(t), \\
& \mathrm{d} y(t)=y(t)\left(r_{2}(r(t))\right. \\
& \quad+\frac{\gamma(r(t)) x(t)}{a(r(t))+m(r(t)) y(t)+n(r(t)) x(t)} \\
& \left.-b_{2}(r(t)) y(t)-\beta_{2}(r(t)) c_{e}^{*}(t)\right) \mathrm{d} t+\sigma_{2}(r(t)) \\
& \cdot y(t) \mathrm{d} B_{2}(t), \\
& x(0)=x_{0}, \\
& y(0)=y_{0}, \\
& r(0)=r_{0} .
\end{aligned}
$$

In order to obtain the threshold conditions of persistence and extinction of system (44), we assume that

$$
\begin{aligned}
& \left(A_{1}\right): \eta_{1}=\sum_{k=1}^{m} \pi_{k}\left(r_{1}(k)-(1 / 2) \sigma_{1}^{2}(k)-c_{\min } \beta_{1}(k)\right)<0, \\
& \left(A_{2}\right): \eta_{2}=\sum_{k=1}^{m} \pi_{k}\left(r_{2}(k)+\gamma(k) / n(k)-(1 / 2) \sigma_{2}^{2}(k)-\right. \\
& \left.c_{\min } \beta_{2}(k)\right)<0, \\
& \left(A_{3}\right): \eta_{3}=\sum_{k=1}^{m} \pi_{k}\left(r_{2}(k)-(1 / 2) \sigma_{2}^{2}(k)-c_{\min } \beta_{2}(k)\right)<0, \\
& \left(A_{4}\right): \eta_{4}=\sum_{k=1}^{m} \pi_{k}\left(r_{1}(k)-\lambda(k) / m(k)-(1 / 2) \sigma_{1}^{2}(k)-\right. \\
& \left.c_{\max } \beta_{1}(k)\right)>0, \\
& \left(A_{5}\right): \eta_{5}=\sum_{k=1}^{m} \pi_{k}\left(r_{1}(k)-(1 / 2) \sigma_{1}^{2}(k)-c_{\max } \beta_{1}(k)\right)> \\
& 0 .
\end{aligned}
$$

Theorem 8. Given initial value $(x(0), y(0), r(0)) \in \mathbb{R}_{+}^{2} \times \mathbb{S}$ for system (44), then

(i) if $\left(A_{1}\right)$ is established, the prey population will be extinct,

(ii) if $\left(A_{2}\right)$ is established, the predator population will be extinct,

(iii) if $\left(A_{1}\right)$ and $\left(A_{3}\right)$ are established, both the prey and the predator will die out. 
Proof. (i) By Itô's formula, we get

$$
\begin{aligned}
& \mathrm{d} \ln x(t)=\left(r_{1}(r(t))-b_{1}(r(t)) x(t)\right. \\
& -\frac{\lambda(r(t)) y(t)}{a(r(t))+m(r(t)) y(t)+n(r(t)) x(t)} \\
& \left.-\frac{\sigma_{1}^{2}(r(t))}{2}-\beta_{1}(r(t)) c_{e}^{*}(t)\right) \mathrm{d} t \\
& +\sigma_{1}(r(t)) \mathrm{d} B_{1}(t) \leq\left(r_{1}(r(t))-\frac{\sigma_{1}^{2}(r(t))}{2}\right. \\
& \left.-\beta_{1}(r(t)) c_{\min }\right) \mathrm{d} t+\sigma_{1}(r(t)) \mathrm{d} B_{1}(t) ;
\end{aligned}
$$

then,

$$
\begin{aligned}
& \frac{\ln x(t)-\ln x(0)}{t} \\
& \leq \frac{1}{t} \int_{0}^{t}\left(r_{1}(r(t))-\frac{\sigma_{1}^{2}(r(t))}{2}-\beta_{1}(r(t)) c_{\min }\right) \mathrm{d} t \\
& \quad+\frac{1}{t} \int_{0}^{t} \sigma_{1}(r(t)) \mathrm{d} B_{1}(t) .
\end{aligned}
$$

By the ergodic theory of the Markov chain and the strong law of large number, we have

$$
\begin{aligned}
& \limsup _{t \rightarrow \infty} \frac{\ln x(t)}{t} \\
& \quad \leq \sum_{k=1}^{m} \pi_{k}\left(r_{1}(k)-\frac{1}{2} \sigma_{1}^{2}(k)-c_{\min } \beta_{1}(k)\right)=\eta_{1},
\end{aligned}
$$

a.s.;

from $\left(A_{1}\right)$, we know

$$
\lim _{t \rightarrow \infty} x(t)=0 . \quad \text { a.s. }
$$

(ii) Similarly, from the second equation of system (44), we have

$$
\begin{aligned}
& \mathrm{d} \ln y(t)=\left(r_{2}(r(t))-b_{2}(r(t)) y(t)\right. \\
& +\frac{\gamma(r(t)) x(t)}{a(r(t))+m(r(t)) y(t)+n(r(t)) x(t)} \\
& \left.\quad-\frac{\sigma_{2}^{2}(r(t))}{2}-\beta_{2}(r(t)) c_{e}^{*}(t)\right) \mathrm{d} t \\
& +\sigma_{2}(r(t)) \mathrm{d} B_{2}(t) ;
\end{aligned}
$$

then,

$$
\begin{aligned}
& \frac{\ln y(t)-\ln y(0)}{t} \leq \frac{1}{t} \int_{0}^{t}\left(r_{2}(r(t))+\frac{\gamma(r(t))}{n(r(t))}\right. \\
& \left.-\frac{\sigma_{2}^{2}(r(t))}{2}-\beta_{2}(r(t)) c_{\min }\right) \mathrm{d} t+\frac{1}{t} \\
& \cdot \int_{0}^{t} \sigma_{2}(r(t)) \mathrm{d} B_{2}(t) ;
\end{aligned}
$$

further,

$$
\begin{aligned}
& \limsup _{t \rightarrow \infty} \frac{\ln y(t)}{t} \\
& \quad \leq \sum_{k=1}^{m} \pi_{k}\left(r_{2}(k)+\frac{\gamma(k)}{n(k)}-\frac{1}{2} \sigma_{2}^{2}(k)-c_{\min } \beta_{2}(k)\right) \\
& \quad=\eta_{2}, \quad \text { a.s.; }
\end{aligned}
$$

from $\left(A_{2}\right)$, we know

$$
\lim _{t \rightarrow \infty} y(t)=0 . \quad \text { a.s. }
$$

(iii) By the condition $\left(A_{1}\right)$, one can get $\lim _{t \rightarrow \infty} x(t)=0$ a.s., so that the limit system of the second equation of system (44) is

$$
\begin{aligned}
& \mathrm{d} y(t)=y(t) \\
& \cdot\left(r_{2}(r(t))-b_{2}(r(t)) y(t)-\beta_{2}(r(t)) c_{e}^{*}(t)\right) \mathrm{d} t \\
& +\sigma_{2}(r(t)) y(t) \mathrm{d} B_{2}(t) \leq y(t) \\
& \cdot\left(r_{2}(r(t))-b_{2}(r(t)) y(t)-\beta_{2}(r(t)) c_{\min }\right) \mathrm{d} t \\
& +\sigma_{2}(r(t)) y(t) \mathrm{d} B_{2}(t) .
\end{aligned}
$$

Through Lemma 2, if $\left(A_{3}\right)$ holds, we obtain

$$
\lim _{t \rightarrow \infty} y(t)=0 . \quad \text { a.s. }
$$

This completes the proof of the theorem.

Remark 9. If $r_{i}(k)(i=1,2)$ remains unchanged and $\sigma_{i}(k)(i=1,2)$ or $c_{\min }$ increases so that $\eta_{1}<0$ or $\eta_{2}<0$, then condition $\left(A_{1}\right)$ or $\left(A_{2}\right)$ is established. That is to say, if the intrinsic growth rate and the predation intensity are relatively fixation, the increase of white noise intensity or pollutant concentration will lead to the extinction of the biological population.

Next, we will discuss the persistence of system (44). Applying Itô's formula to the first equation of system (44), one can get

$$
\begin{aligned}
& \mathrm{d} \ln x(t) \geq\left(r_{1}(r(t))-b_{1}(r(t)) x(t)-\frac{\lambda(r(t))}{m(r(t))}\right. \\
& \left.-\frac{\sigma_{1}^{2}(r(t))}{2}-\beta_{1}(r(t)) c_{\max }\right) \mathrm{d} t \\
& +\sigma_{1}(r(t)) \mathrm{d} B_{1}(t)
\end{aligned}
$$


then,

$$
\begin{aligned}
& \frac{\ln x(t)-\ln x(0)}{t} \geq \frac{1}{t} \int_{0}^{t}\left(r_{1}(r(t))-b_{1}(r(t)) x(t)\right. \\
& \left.-\frac{\lambda(r(t))}{m(r(t))}-\frac{\sigma_{1}^{2}(r(t))}{2}-\beta_{1}(r(t)) c_{\max }\right) \mathrm{d} t \\
& +\frac{1}{t} \int_{0}^{t} \sigma_{1}(r(t)) \mathrm{d} B_{1}(t),
\end{aligned}
$$

when $t$ is large enough, we derive

$$
\begin{aligned}
& \frac{\ln x(t)}{t} \\
& \geq \sum_{k=1}^{m} \pi_{k}\left(r_{1}(k)-\frac{\lambda(k)}{m(k)}-\frac{1}{2} \sigma_{1}^{2}(k)-c_{\max } \beta_{1}(k)\right) \\
& \quad-\frac{\check{b}_{1}}{t} \int_{0}^{t} x(t) \mathrm{d} t-\varepsilon,
\end{aligned}
$$

where $\varepsilon$ is a sufficiently small positive number. In view of $\left(A_{4}\right)$ and Lemma 4 , we deduce

$$
\liminf _{t \rightarrow \infty} \frac{1}{t} \int_{0}^{t} x(t) \mathrm{d} t \geq \frac{\eta_{4}}{\check{b}_{1}}, \quad \text { a.s. }
$$

That is, the prey population of system (44) will be persistence in mean under condition $\left(A_{4}\right)$.

Furthermore, the persistent property of the predator species of system (44) can be investigated as follows.

From the first equation of system (44), we have

$$
\begin{aligned}
& \mathrm{d} x(t) \leq x(t) \\
& \quad \cdot\left(r_{1}(r(t))-b_{1}(r(t)) x(t)-\beta_{1}(r(t)) c_{e}^{*}(t)\right) \mathrm{d} t \\
& \quad+\sigma_{1}(r(t)) x(t) \mathrm{d} B_{1}(t) .
\end{aligned}
$$

Consider the following stochastic differential equations:

$$
\begin{aligned}
& \mathrm{d} \phi(t)=\phi(t) \\
& \quad \cdot\left(r_{1}(r(t))-b_{1}(r(t)) \phi(t)-\beta_{1}(r(t)) c_{e}^{*}(t)\right) \mathrm{d} t \\
& \quad+\sigma_{1}(r(t)) \phi(t) \mathrm{d} B_{1}(t), \\
& \phi(0)=x(0), \\
& \mathrm{d} \psi(t)=\psi(t) \\
& \quad \cdot\left(r_{1}(r(t))-b_{1}(r(t)) \psi(t)-\beta_{1}(r(t)) c_{\max }\right) \mathrm{d} t \\
& \quad+\sigma_{1}(r(t)) \psi(t) \mathrm{d} B_{1}(t), \\
& \psi(0)=x(0) .
\end{aligned}
$$

Obviously, $x(t) \leq \phi(t), \psi(t) \leq \phi(t)$. Using Lemma 2 , if $\eta_{5}>$ 0 , system (61) is ergodic and there exists a unique stationary distribution $\mu_{\psi}$, such that

$$
\begin{aligned}
\sum_{k=1}^{m} b_{1}(k) \int_{\mathbb{R}^{+}} x \mu_{\psi}(\mathrm{d} x, k) \\
\quad=\sum_{k=1}^{m} \pi_{k}\left(r_{1}(k)-\frac{1}{2} \sigma_{1}^{2}(k)-c_{\max } \beta_{1}(k)\right) .
\end{aligned}
$$

Applying Itô's formula to (60) and then integrating from 0 to $t$, we get

$$
\begin{aligned}
& \frac{\ln \phi(t)-\ln \phi(0)}{t}=\frac{1}{t} \int_{0}^{t}\left(r_{1}(r(t))-\frac{\sigma_{1}^{2}(r(t))}{2}\right. \\
& \left.-\beta_{1}(r(t)) c_{e}^{*}(t)-b_{1}(r(t)) \phi(t)\right) \mathrm{d} t+\frac{1}{t} \\
& \cdot \int_{0}^{t} \sigma_{1}(r(t)) \mathrm{d} B_{1}(t) .
\end{aligned}
$$

From the first equation of system (44), it yields that

$$
\begin{aligned}
& \frac{\ln x(t)-\ln x(0)}{t} \\
& =\frac{1}{t} \int_{0}^{t}\left(r_{1}(r(t))-\frac{\sigma_{1}^{2}(r(t))}{2}-\beta_{1}(r(t)) c_{e}^{*}(t)\right) \mathrm{d} t \\
& \quad-\frac{1}{t} \int_{0}^{t} \frac{\lambda(r(t)) y(t)}{a(r(t))+m(r(t)) y(t)+n(r(t)) x(t)} \mathrm{d} t \\
& \quad-\frac{1}{t} \int_{0}^{t} b_{1}(r(t)) x(t) \mathrm{d} t+\frac{1}{t} \int_{0}^{t} \sigma_{1}(r(t)) \mathrm{d} B_{1}(t) .
\end{aligned}
$$

Due to $x(t) \leq \phi(t)$, one can get

$$
\frac{1}{t} \int_{0}^{t}(\phi(t)-x(t)) \mathrm{d} t \leq \frac{1}{t} \int_{0}^{t} \frac{\check{\lambda}}{\widehat{a} \widehat{b}_{1}} y(t) \mathrm{d} t . \quad \text { a.s. }
$$

From the second equation of system (44) we have

$$
\begin{aligned}
& \frac{\ln y(t)}{t}=\frac{\ln y(0)}{t}+\frac{1}{t} \int_{0}^{t}\left(r_{2}(r(t))-\frac{\sigma_{2}^{2}(r(t))}{2}\right. \\
& \left.-\beta_{2}(r(t)) c_{e}^{*}(t)\right) \mathrm{d} t-\frac{1}{t} \int_{0}^{t} b_{2}(r(t)) y(t) \mathrm{d} t \\
& +\frac{1}{t} \int_{0}^{t} \frac{\gamma(r(t)) \phi(t)}{a(r(t))+n(r(t)) \phi(t)} \mathrm{d} t-\frac{1}{t}
\end{aligned}
$$




$$
\begin{aligned}
& \cdot \int_{0}^{t}\left(\frac{\gamma(r(t)) \phi(t)}{a(r(t))+n(r(t)) \phi(t)}\right) \mathrm{d} t-\frac{1}{t} \\
& -\frac{\gamma(r(t)) x(t)}{a(r(t))+n(r(t)) x(t)} \\
& \cdot \int_{0}^{t}\left(\frac{\gamma(r(t)) x(t)}{a(r(t))+n(r(t)) x(t)}\right. \\
& \left.-\frac{\gamma(r(t)) x(t)}{a(r(t))+m(r(t)) y(t)+n(r(t)) x(t)}\right) \mathrm{d} t \\
& +\frac{1}{t} \int_{0}^{t} \sigma_{2}(r(t)) \mathrm{d} B_{2}(t) \geq \frac{\ln y(0)}{t}+\frac{1}{t} \\
& \cdot \int_{0}^{t}\left(r_{2}(r(t))-\frac{\sigma_{2}^{2}(r(t))}{2}-\beta_{2}(r(t)) c_{\max }\right) \mathrm{d} t \\
& +\frac{1}{t} \int_{0}^{t} \frac{\gamma(r(t)) \psi(t)}{a(r(t))+n(r(t)) \psi(t)} \mathrm{d} t-\left(\check{b}_{2}+\frac{\check{\gamma} \check{\lambda}}{\widehat{a}^{2} \widehat{b}_{1}}\right. \\
& +\frac{\check{\gamma} \check{m}}{\widehat{a} \widehat{n}} \frac{1}{t} \int_{0}^{t} y(t) \mathrm{d} t+\frac{1}{t} \int_{0}^{t} \sigma_{2}(r(t)) \mathrm{d} B_{2}(t) .
\end{aligned}
$$

By Lemma 4, if

$$
\begin{aligned}
& \left(A_{6}\right): \eta_{6}=\sum_{k=1}^{m} \pi_{k}\left(r_{2}(k)-(1 / 2) \sigma_{2}^{2}(k)-c_{\max } \beta_{2}(k)\right)+ \\
& \sum_{k=1}^{m} \pi_{k} \int_{\mathbb{R}^{+}}(\gamma(k) x /(a(k)+n(k) x)) \mu_{\psi}(\mathrm{d} x, k)>0
\end{aligned}
$$

holds, then

$$
\liminf _{t \rightarrow \infty} \frac{1}{t} \int_{0}^{t} y(t) \mathrm{d} t \geq \frac{\eta_{6}}{\check{b}_{2}+\check{\gamma} \check{\lambda} / \widehat{a}^{2} \widehat{b}_{1}+\check{\gamma} \check{m} / \widehat{a} \widehat{n}} \quad \text { a.s. }
$$

In summary, one gets the following.

Theorem 10. Given initial value $(x(0), y(0), r(0)) \in \mathbb{R}_{+}^{2} \times \mathbb{S}$ for system (44), then

(i) if $\left(A_{4}\right)$ is established, the prey population will be persistent in mean,

(ii) if conditions $\left(A_{5}\right)$ and $\left(A_{6}\right)$ are satisfied, the predator population will be persistent in mean.

Remark 11. (i) It can be seen from $\eta_{4}$, in the case where the intrinsic growth rate and the predation intensity are relatively constant, only by reducing the intensity of white noise or pollutant concentration, so that $\left(A_{4}\right)$ can be established to ensure the lasting survival of the prey population.

(ii) Obviously, $\eta_{4}<\eta_{5}$; if the prey population is persistent, the predator population is persistent as long as the white noise interference intensity or the toxin concentration is small enough, such that $\left(A_{6}\right)$ is established. As can be seen from condition $\left(A_{6}\right)$, the omnivorous nature of $y$ contributes to its permanence.

In system (4), if the concentration of the toxicant in the environment remains unchanged, that is, $c_{e}(t)=c$ is a positive constant, then the system can be converted into the following system:

$$
\begin{aligned}
& \mathrm{d} x(t)=x(t)\left(r_{1}(r(t))-b_{1}(r(t)) x(t)\right. \\
& -\frac{\lambda(r(t)) y(t)}{a(r(t))+m(r(t)) y(t)+n(r(t)) x(t)} \\
& \left.-c \beta_{1}(r(t))\right) \mathrm{d} t+\sigma_{1}(r(t)) x(t) \mathrm{d} B_{1}(t), \\
& \mathrm{d} y(t)=y(t)\left(r_{2}(r(t))\right. \\
& +\frac{\gamma(r(t)) x(t)}{a(r(t))+m(r(t)) y(t)+n(r(t)) x(t)} \\
& \left.-b_{2}(r(t)) y(t)-c \beta_{2}(r(t))\right) \mathrm{d} t+\sigma_{2}(r(t)) \\
& \cdot y(t) \mathrm{d} B_{2}(t), \\
& x(0)=x_{0}, \\
& y(0)=y_{0}, \\
& r(0)=r_{0} .
\end{aligned}
$$

Lemma 12 (see [77]). System (7) is ergodic and positive recurrent if the following conditions are satisfied:

(i) For $i \neq j, q_{i j}>0$.

(ii) For each $k \in \mathbb{S}$,

$$
\sigma\|\xi\|^{2} \leq \xi^{T} G(x, k) \xi \leq \sigma^{-1}\|\xi\|^{2}
$$

for all $\xi \in \mathbb{R}^{n}$, with some constant $\sigma \in(0,1]$ for all $x \in \mathbb{R}^{n}$.

(iii) There exists a bounded open set $\mathscr{D} \subset \mathbb{R}^{n}$ with a smooth boundary satisfying that, for each $k \in \mathbb{S}$, there is a twice continuously differentiable nonnegative function $V(\cdot, \cdot)$ : $\mathscr{D}^{c} \rightarrow \mathbb{R}$ and that for some $\zeta>0, \mathscr{L} V(x, k) \leq-\zeta$, for any $(x, k) \in \mathscr{D}^{c} \times \mathbb{S}$.

Moreover, the Markov process $(x(t), r(t))$ has a unique ergodic stationary distribution $\mu(\cdot, \cdot)$. Hence, for any Borel measurable function $H(\cdot, \cdot): \mathbb{R}^{n} \times \mathbb{S} \rightarrow \mathbb{R}$, if $\sum_{k \in \mathbb{S}} \int_{\mathbb{R}^{n}}|H(x, k)| \mu(x, k) \mathrm{d} x<\infty$, then

$$
\begin{aligned}
& P\left(\lim _{t \rightarrow \infty} \frac{1}{t} \int_{0}^{t} H(x(s), r(s)) \mathrm{d} s\right. \\
& \left.\quad=\sum_{k \in \mathbb{S}} \int_{\mathbb{R}^{n}} H(x, k) \mu(x, k) \mathrm{d} x\right)=1 .
\end{aligned}
$$

Recently, the ergodicity and stationary distribution have been explored by many authors. In the following, we give sufficient conditions for the existence of stationary distribution of system (68) and prove the following theorem by showing that system (68) satisfies the three conditions in Lemma 12. 
Theorem 13. Assume that for $i \neq j, q_{i j}>0$ and

$$
\begin{aligned}
& \left(B_{1}\right): \sum_{k \in \mathbb{S}} \pi_{k}\left(r_{1}(k)-\lambda(k) / m(k)-\sigma_{1}^{2}(k) / 2-c \beta_{1}(k)\right)> \\
& 0, \\
& \left(B_{2}\right): \sum_{k \in \mathbb{S}} \pi_{k}\left(r_{2}(k)-\sigma_{2}^{2}(k) / 2-c \beta_{2}(k)\right)>0
\end{aligned}
$$

hold; then the stochastic process $(x(t), y(t), r(t))$ of system (68) is ergodic and has a unique stationary distribution in $\mathbb{R}_{+}^{2} \times \mathbb{S}$.

Proof. By the assumption $q_{i j}>0$ for $i \neq j$ in Theorem 13, condition (i) in Lemma 12 is satisfied. Let $g(x, y, k)=$ $\operatorname{diag}\left(\sigma_{1}(k) x, \sigma_{2}(k) y\right)$; then

$$
\begin{aligned}
G(x, y, k) & =g(x, y, k) g^{T}(x, y, k) \\
& =\operatorname{diag}\left(\sigma_{1}^{2}(k) x^{2}, \sigma_{2}^{2}(k) y^{2}\right) .
\end{aligned}
$$

Define a bounded open subset as follows:

$$
U=\left(\varepsilon, \frac{1}{\varepsilon}\right) \times\left(\varepsilon, \frac{1}{\varepsilon}\right) \subset \mathbb{R}_{+}^{2},
$$

where $0<\varepsilon<1$ is a constant. Let $\sigma=\min \left\{1, M_{1}, 1 / M_{2}\right\}$; here $M_{1}=\min _{(x, y, k) \in \bar{U} \times \mathbb{S}}\left\{\sigma_{1}^{2}(k) x^{2}+\sigma_{2}^{2}(k) y^{2}\right\}$ and $M_{2}=$ $\max _{(x, y, k) \in \bar{U} \times \mathbb{S}}\left\{\sigma_{1}^{2}(k) x^{2}+\sigma_{2}^{2}(k) y^{2}\right\}$. For $(x, y, k) \in U \times \mathbb{S}$, we have

$$
\begin{aligned}
\sigma\|\xi\|^{2} & \leq \xi^{T} G(x, y, k) \xi=\sigma_{1}^{2}(k) x^{2} \xi_{1}^{2}+\sigma_{2}^{2}(k) y^{2} \xi_{2}^{2} \\
& \leq \sigma^{-1}\|\xi\|^{2}
\end{aligned}
$$

for all $\xi \in \mathbb{R}^{2}$. Thus condition (ii) in Lemma 12 holds. Therefore, it remains for us to verify condition (iii) in Lemma 12.

Define a $C^{2}$-function on $\mathbb{R}_{+}^{2} \times \mathbb{S}$,

$$
\begin{aligned}
V(x, y, k)= & \left(1-v \eta_{k}\right) x^{-v}+\left(1-v \delta_{k}\right) y^{-v} \\
& +(x+2)(y+M)=: V_{4}+V_{5},
\end{aligned}
$$

where $V_{4}=\left(1-v \eta_{k}\right) x^{-v}+\left(1-v \delta_{k}\right) y^{-v}, V_{5}=(x+2)(y+$ $M), v$ is a sufficiently small positive constant satisfying $v<$ $\min \left(1 / \eta_{k}, 1 / \delta_{k}\right)$, and $M \geq 1+\left(1 / 4 \widehat{b}_{1} \widehat{b}_{2}\right)\left(\check{r}_{1}-c \widehat{\beta}_{1}+\check{r}_{2}-c \widehat{\beta}_{2}+\right.$ $\check{\gamma} / \widehat{n})^{2} . \eta_{k}, \delta_{k}$ are quantities to be determined below.

An application of the operator $\mathscr{L}$ to $V_{4}$ yields

$$
\begin{aligned}
& \mathscr{L} V_{4}(x, y, k)=-v\left(1-v \eta_{k}\right) x^{-v}\left(r_{1}(k)-b_{1}(k) x\right. \\
& \left.-\frac{\lambda(k) y}{a(k)+m(k) y+n(k) x}-c \beta_{1}(k)\right)+\frac{1}{2} v(1 \\
& \quad+v)\left(1-v \eta_{k}\right) \sigma_{1}^{2}(k) x^{-v}-v \sum_{i \neq k} q_{k i}\left(\eta_{i}-\eta_{k}\right) x^{-v} \\
& -v\left(1-v \delta_{k}\right) y^{-v}\left(r_{2}(k)\right.
\end{aligned}
$$

$$
\begin{aligned}
& \left.+\frac{\gamma(k) x}{a(k)+m(k) y+n(k) x}-b_{2}(k) y-c \beta_{2}(k)\right) \\
& +\frac{1}{2} v(1+v)\left(1-v \delta_{k}\right) \sigma_{2}^{2}(k) y^{-v} \\
& -v \sum_{i \neq k} q_{k i}\left(\delta_{i}-\delta_{k}\right) y^{-v} \leq v\left(1-v \eta_{k}\right) \\
& \cdot\left[-\left(r_{1}(k)-\frac{\lambda(k)}{m(k)}-c \beta_{1}(k)-\frac{\sigma_{1}^{2}(k)}{2}\right)\right. \\
& -\sum_{i \neq k} q_{k i}\left(\eta_{i}-\eta_{k}\right)+\frac{1}{2} v \sigma_{1}^{2}(k) \\
& \left.-\frac{v \eta_{k}}{1-v \eta_{k}} \sum_{i \neq k} q_{k i}\left(\eta_{i}-\eta_{k}\right)\right] x^{-v}+v\left(1-v \delta_{k}\right) \\
& \cdot\left[-\left(r_{2}(k)-c \beta_{2}(k)-\frac{1}{2} \sigma_{2}^{2}(k)\right)-\sum_{i \neq k} q_{k i}\left(\delta_{i}-\delta_{k}\right)\right. \\
& \left.+\frac{1}{2} v \sigma_{2}^{2}(k)-\frac{v \delta_{k}}{1-v \delta_{k}} \sum_{i \neq k} q_{k i}\left(\delta_{i}-\delta_{k}\right)\right] y^{-v}+v(1 \\
& \left.-v \eta_{k}\right) b_{1}(k) x^{1-v}+v\left(1-v \delta_{k}\right) b_{2}(k) y^{1-v} .
\end{aligned}
$$

Define the vectors $\xi=\left(\xi_{1}, \xi_{2}, \ldots, \xi_{m}\right)^{T}$ and $\mathcal{\vartheta}=\left(\vartheta_{1}\right.$, $\left.\vartheta_{2}, \ldots, \vartheta_{m}\right)^{T}$ with $\xi_{k}=-\left(r_{1}(k)-\lambda(k) / m(k)-c \beta_{1}(k)-\right.$ $\left.\left(\sigma_{1}^{2} / 2\right)(k)\right), \vartheta_{k}=-\left(r_{2}(k)-c \beta_{2}(k)-(1 / 2) \sigma_{2}^{2}(k)\right)$. As the generator matrix $\Gamma$ is irreducible, for each $\xi_{k}$ and $\vartheta_{k}$, there exists $\eta=\left(\eta_{1}, \eta_{2}, \ldots, \eta_{m}\right)^{T}$ and $\delta=\left(\delta_{1}, \delta_{2}, \ldots, \delta_{m}\right)^{T}$, respectively, which is a solution of the Poisson system [78]

$$
\begin{gathered}
(\Gamma \eta)_{k}-\xi_{k}=-\sum_{j=1}^{m} \pi_{j} \xi_{j}, \\
(\Gamma \delta)_{k}-\vartheta_{k}=-\sum_{j=1}^{m} \pi_{j} \vartheta_{j} .
\end{gathered}
$$

Therefore we have

$$
\begin{aligned}
& -\left(r_{1}(k)-\frac{\lambda(k)}{m(k)}-c \beta_{1}(k)-\frac{\sigma_{1}^{2}}{2}(k)\right) \\
& -\sum_{i \neq k} q_{k i}\left(\eta_{i}-\eta_{k}\right) \\
& =-\sum_{k \in \mathbb{S}} \pi_{k}\left(r_{1}(k)-\frac{\lambda(k)}{m(k)}-\frac{\sigma_{1}^{2}(k)}{2}-c \beta_{1}(k)\right), \\
& -\left(r_{2}(k)-c \beta_{2}(k)-\frac{1}{2} \sigma_{2}^{2}(k)\right)-\sum_{i \neq k} q_{k i}\left(\delta_{i}-\delta_{k}\right) \\
& =-\sum_{k \in \mathbb{S}} \pi_{k}\left(r_{2}(k)-\frac{\sigma_{2}^{2}(k)}{2}-c \beta_{2}(k)\right) .
\end{aligned}
$$


Combining (75), (77), and (78), we obtain

$$
\begin{aligned}
& \mathscr{L} V_{4}(x, y, k) \leq v\left(1-v \eta_{k}\right) \\
& .\left[-\sum_{k \in \mathbb{S}} \pi_{k}\left(r_{1}(k)-\frac{\lambda(k)}{m(k)}-\frac{\sigma_{1}^{2}(k)}{2}-c \beta_{1}(k)\right)\right. \\
& \left.+\frac{1}{2} v \sigma_{1}^{2}(k)-\frac{v \eta_{k}}{1-v \eta_{k}} \sum_{i \neq k} q_{k i}\left(\eta_{i}-\eta_{k}\right)\right] x^{-v}+v(1 \\
& \left.-v \delta_{k}\right)\left[-\sum_{k \in \mathbb{S}} \pi_{k}\left(r_{2}(k)-\frac{\sigma_{2}^{2}(k)}{2}-c \beta_{2}(k)\right)\right. \\
& \left.+\frac{1}{2} v \sigma_{2}^{2}(k)-\frac{v \delta_{k}}{1-v \delta_{k}} \sum_{i \neq k} q_{k i}\left(\delta_{i}-\delta_{k}\right)\right] y^{-v}+v(1 \\
& \left.-v \eta_{k}\right) b_{1}(k) x^{1-v}+v\left(1-v \delta_{k}\right) b_{2}(k) y^{1-v} .
\end{aligned}
$$

Similarly, for $V_{5}(x, y)$, we calculate

$$
\begin{aligned}
& \mathscr{L} V_{5}=(y+M) x\left(r_{1}(k)-b_{1}(k) x\right. \\
& \left.-\frac{\lambda(k) y}{a(k)+m(k) y+n(k) x}-c \beta_{1}(k)\right)+(x+2) \\
& \cdot y\left(r_{2}(k)+\frac{\gamma(k) x}{a(k)+m(k) y+n(k) x}-b_{2}(k) y\right. \\
& \left.-c \beta_{2}(k)\right) \leq-\widehat{b}_{1} x^{2}+M\left(\check{r}_{1}-c \widehat{\beta}_{1}\right) x-\widehat{b}_{2} y^{2} \\
& +2\left(\check{r}_{2}-c \widehat{\beta}_{2}+\frac{\check{\gamma}}{\widehat{n}}\right) y .
\end{aligned}
$$

From conditions $\left(B_{1}\right)$ and $\left(B_{2}\right)$, we can choose $v$ sufficiently small such that

$$
\begin{aligned}
& -\sum_{k \in \mathbb{S}} \pi_{k}\left(r_{1}(k)-\frac{\lambda(k)}{m(k)}-\frac{\sigma_{1}^{2}(k)}{2}-c \beta_{1}(k)\right) \\
& +\frac{1}{2} v \sigma_{1}^{2}(k)-\frac{v \eta_{k}}{1-v \eta_{k}} \sum_{i \neq k} q_{k i}\left(\eta_{i}-\eta_{k}\right)<0, \\
& -\sum_{k \in \mathbb{S}} \pi_{k}\left(r_{2}(k)-\frac{\sigma_{2}^{2}(k)}{2}-c \beta_{2}(k)\right)+\frac{1}{2} v \sigma_{2}^{2}(k) \\
& -\frac{v \delta_{k}}{1-v \delta_{k}} \sum_{i \neq k} q_{k i}\left(\delta_{i}-\delta_{k}\right)<0 .
\end{aligned}
$$

So $\mathscr{L} V=\mathscr{L} V_{4}+\mathscr{L} V_{5}$ can be estimated as follows:

$$
\begin{aligned}
& \mathscr{L} V \leq v\left(1-v \eta_{k}\right) \\
& \cdot\left[-\sum_{k \in \mathbb{S}} \pi_{k}\left(r_{1}(k)-\frac{\lambda(k)}{m(k)}-\frac{\sigma_{1}^{2}(k)}{2}-c \beta_{1}(k)\right)\right.
\end{aligned}
$$

$$
\begin{aligned}
& \left.+\frac{1}{2} v \sigma_{1}^{2}(k)-\frac{v \eta_{k}}{1-v \eta_{k}} \sum_{i \neq k} q_{k i}\left(\eta_{i}-\eta_{k}\right)\right] x^{-v}+v(1 \\
& \left.-v \eta_{k}\right) b_{1}(k) x^{1-v}-\widehat{b}_{1} x^{2}+M\left(\check{r}_{1}-c \widehat{\beta}_{1}\right) x+v(1 \\
& \left.-v \delta_{k}\right)\left[-\sum_{k \in \mathbb{S}} \pi_{k}\left(r_{2}(k)-\frac{\sigma_{2}^{2}(k)}{2}-c \beta_{2}(k)\right)\right. \\
& \left.+\frac{1}{2} v \sigma_{2}^{2}(k)-\frac{v \delta_{k}}{1-v \delta_{k}} \sum_{i \neq k} q_{k i}\left(\delta_{i}-\delta_{k}\right)\right] y^{-v}+v(1 \\
& \left.-v \delta_{k}\right) b_{2}(k) y^{1-v}-\widehat{b}_{2} y^{2}+2\left(\check{r}_{2}-c \widehat{\beta}_{2}+\frac{\check{\gamma}}{\widehat{n}}\right) y \\
& =f_{1}(x)+f_{2}(y) .
\end{aligned}
$$

It is easy to see that

$$
\begin{aligned}
& \mathscr{L} V \leq f_{1}(x)+f_{2}(y) \\
& \leq \begin{cases}f_{1}(x)+f_{2}^{u}(y) \longrightarrow-\infty, & \text { if } x \longrightarrow 0 \text { or } x \longrightarrow+\infty, \\
f_{1}^{u}(x)+f_{2}(y) \longrightarrow-\infty, & \text { if } y \longrightarrow 0 \text { or } y \longrightarrow+\infty .\end{cases}
\end{aligned}
$$

Consequently, we derive that, for a sufficiently small $\varepsilon$,

$$
\mathscr{L} V(x, y, k) \leq-1, \quad \forall(x, y, k) \in U^{c} \times \mathbb{S}
$$

Using Lemma 12, we obtain the conclusion that $(x(t), y(t)$, $r(t))$ is ergodic and positive recurrent; that is, system (68) is positive recurrent and has a unique stationary distribution.

This completes the proof of Theorem 13

\section{Conclusions and Numerical Simulations}

In this article, we discussed the dynamics of stochastic preypredator models with Beddington-DeAngelis functional response in polluted environment.

Firstly, for system (3), there are the following properties:

(1) If $h_{1}<0$ and $h_{2}>0$, the limit system of (3) has a prey extinction periodic solution $\left(0, y^{*}(t)\right)$.

(2) If conditions $\left(H_{1}\right),\left(H_{2}\right)$, and $\left(H_{3}\right)$ are established, the limit system of (3) has a positive periodic solution.

Secondly, system (4) possesses the following properties:

(1) If $\eta_{1}<0$, the prey population $x(t)$ will be extinct.

(2) If $\eta_{2}<0$, the predator population $y(t)$ will be extinct.

(3) If $\eta_{1}<0$ and $\eta_{3}<0$, then prey population and predator population will die out.

(4) If $\eta_{4}>0$, the prey population $x(t)$ will be persistent in mean.

(5) If $\eta_{5}>0$ and $\eta_{6}>0$, the predator population $y(t)$ will be persistent in mean.

To verify the correctness of the theoretical analysis, numerical simulations are employed in the following examples. 


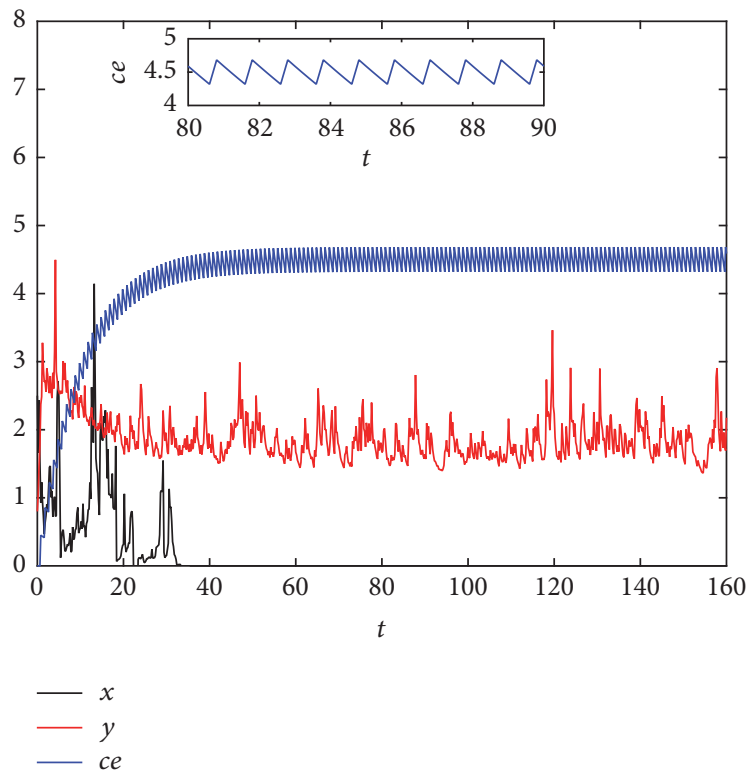

FIGURE 1: Sample paths of $x(t)$ and $y(t)$ with initial conditions $x(0)=2.5$ and $y(0)=0.8$.

Assume that the Markov chain $r(t)$ take values in $\mathbb{S}=$ $\{1,2\}$ with the generator

$$
\Gamma=\left(\begin{array}{cc}
-1 & 1 \\
2 & -2
\end{array}\right)
$$

By the linear equations $\pi \Gamma=0$, we can see $\left(\pi_{1}, \pi_{2}\right)=$ $(2 / 3,1 / 3)$ which is the stationary distribution of $r(t)$. Furthermore, in the following examples, we suppose $\tau=0.5$ and $h=0.1$, consistently.

\subsection{The Existence of Periodic Solutions of System (3)}

Example 14. Assume $r_{1}=1.2, r_{2}=2.3, \beta_{1}=0.18, \beta_{2}=0.22$, $b_{1}=0.5, b_{2}=0.8, \lambda=0.3, \gamma=0.5, a=1.4, m=0.8$, and $n=1.8$.

Case 1. We choose the density of white noise as the following: $\sigma_{1}(t)=0.09+0.8 \sin ((2 \pi / \tau) t), \sigma_{2}(t)=0.1+0.01 \sin ((2 \pi / \tau) t)$, and let $\mu=0.45$.

Note that $h_{1}=-0.5841<0, h_{2}=0.3150>0$. The conditions of Theorem 6 hold, so there exists a boundary periodic solution $\left(0, y^{*}(t)\right)$ of system (3) (see Figure 1).

Case 2. We change the density of the white noise to $\sigma_{1}(t)=$ $0.02+0.2 \sin ((2 \pi / \tau) t), \sigma_{2}(t)=0.07+0.1 \sin ((2 \pi / \tau) t)$, and $\mu=0.13$. This gives $h_{1}=0.7218>0, h_{2}=1.7230>0, \lambda_{1}=$ $0.0634>0$, and $\xi_{2}=0.2024>0$; choose $\xi_{1}=0.1$ according to $\xi_{1}>\left(\gamma^{2} / 4 b_{1} h_{1}\right) \lambda_{1}$ and $\left(b_{2} \xi_{1} / \gamma^{2}\right)\left[-h_{2}+\left(r_{2}+\gamma / n-\beta_{2} c_{\min }\right)\right]<$ $\left(\xi_{2} / a b_{1}\right)\left(r_{1}-\beta_{1} c_{\max }\right)$.

From Theorem 7, we know that there exists a positive $\tau$ periodic solution of system (3) (see Figure 2).
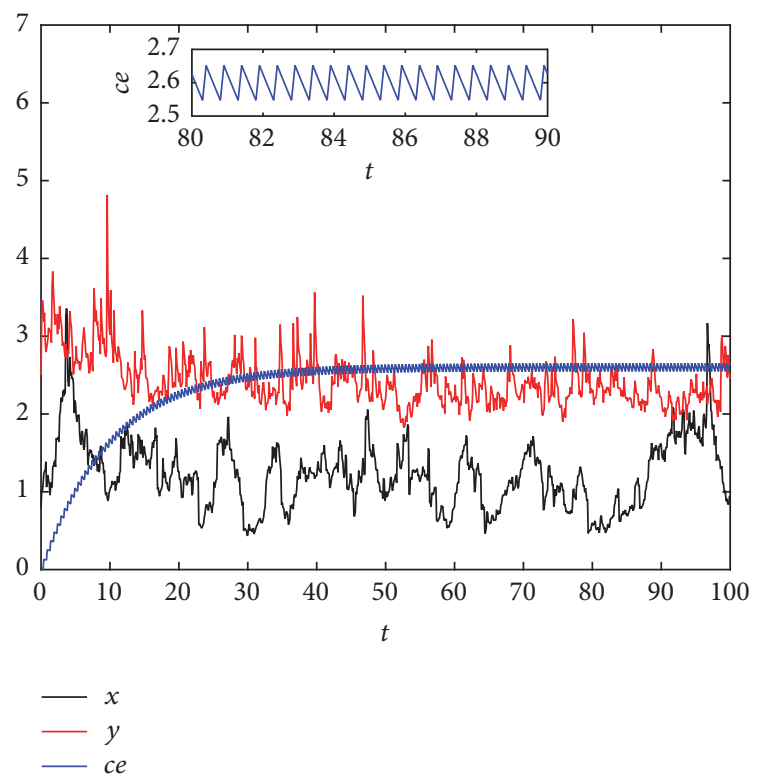

FIGURE 2: Sample paths of $x(t)$ and $y(t)$ with initial conditions $x(0)=0.8$ and $y(0)=2.5$.

\subsection{The Extinction and Persistence of System (4)}

Example 15. Choose parameters $\left(r_{1}(1), r_{2}(1)\right)=(2.3,1.5)$, $\lambda(1)=0.06, \gamma(1)=0.3,\left(b_{1}(1), b_{2}(1)\right)=(1.8,1.6), a(1)=0.4$, $m(1)=0.6, n(1)=0.3$, and $\left(\beta_{1}(1), \beta_{2}(1)\right)=(0.4,0.2)$, if $k=1$, and $\left(r_{1}(2), r_{2}(2)\right)=(2.5,1.3), \lambda(2)=0.08, \gamma(2)=0.2$, $\left(b_{1}(2), b_{2}(2)\right)=(1.8,1.6), a(2)=0.4, m(2)=0.8, n(2)=0.4$, and $\left(\beta_{1}(2), \beta_{2}(2)\right)=(0.5,0.3)$, if $k=2$.

Case 1. Let $\mu=0.15,\left(\sigma_{1}(1), \sigma_{2}(1)\right)=(1.7,2.1)$, and $\left(\sigma_{1}(2), \sigma_{2}(2)\right)=(1.4,0.9)$; we note $\eta_{1}=-0.1911<0$, 

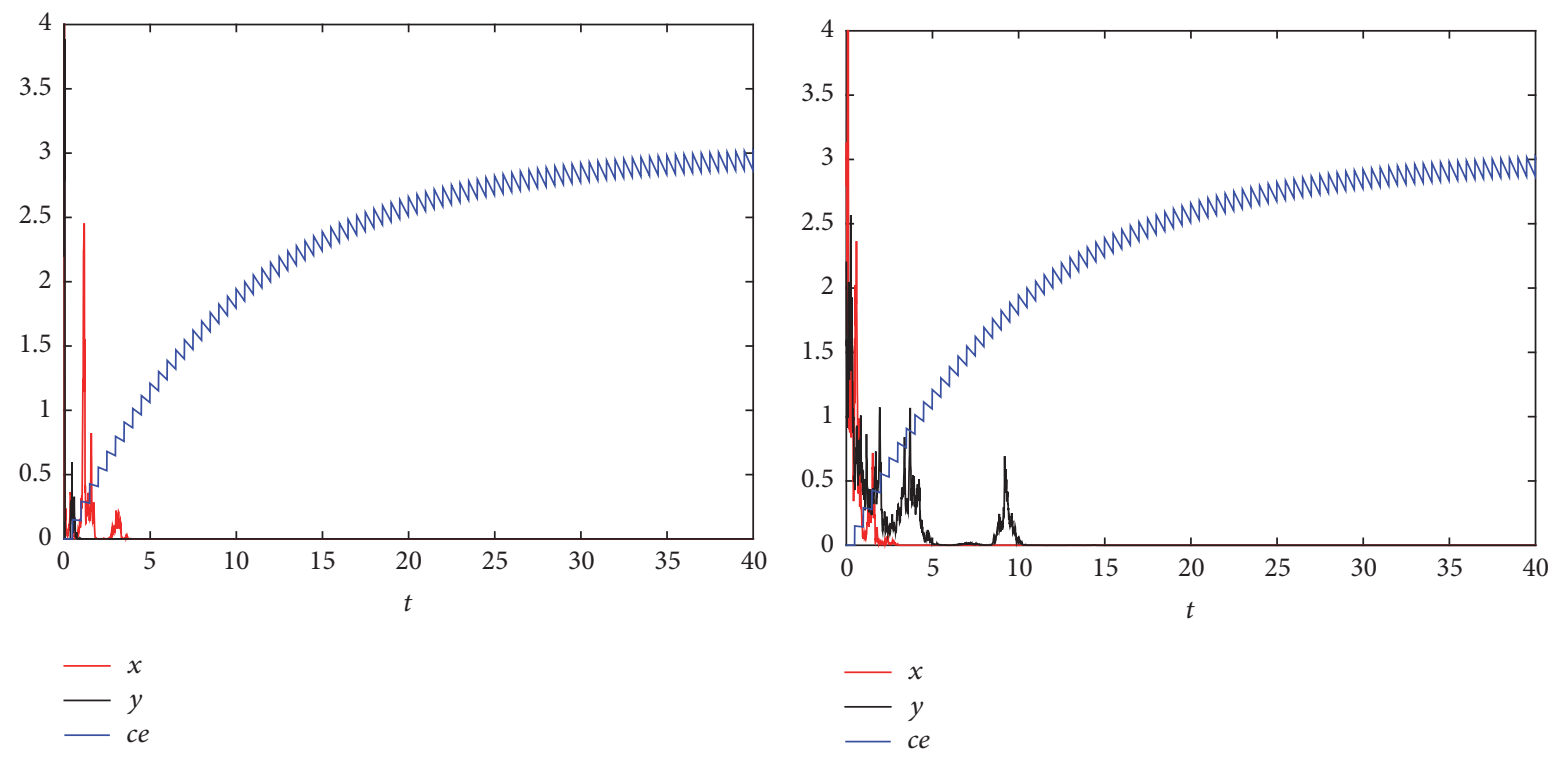

(a) $k=1$

(b) $k=2$

Figure 3: Sample paths of $x(t), y(t)$ with initial value $x(0)=2.8$ and $y(0)=2.2$.

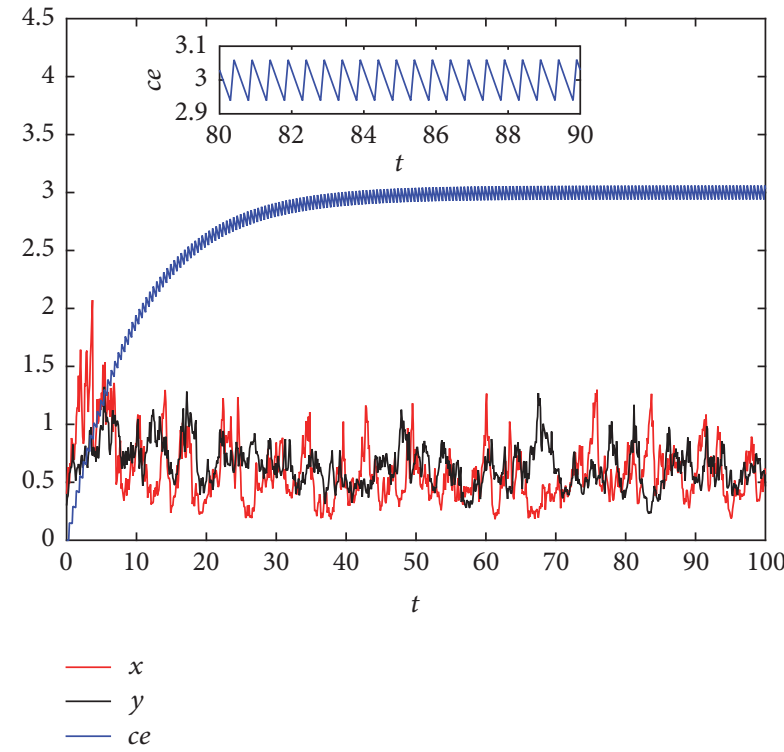

(a) $k=1$

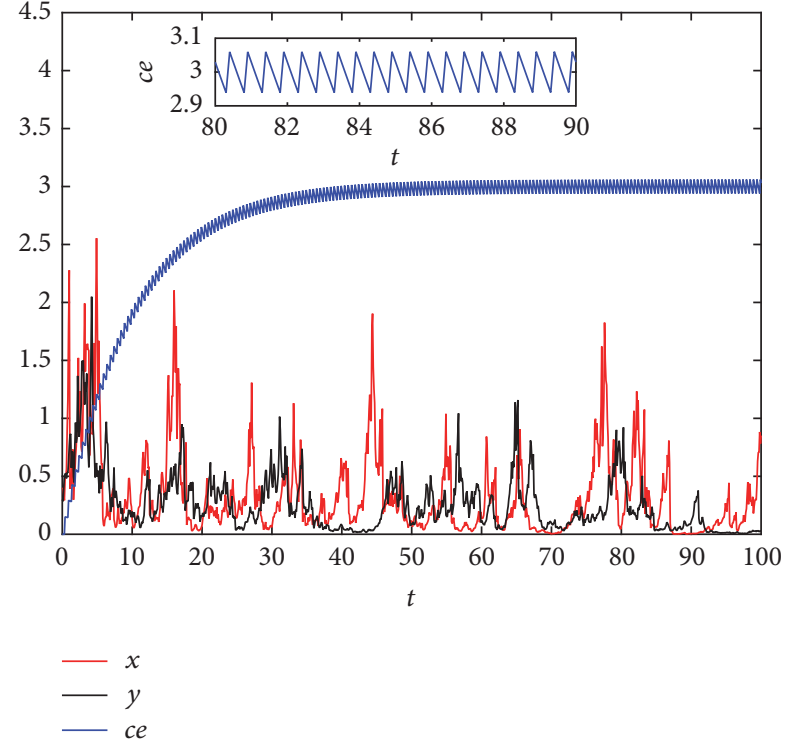

(b) $k=2$

FIGURE 4: Sample paths of $x(t), y(t)$ with initial value $x(0)=0.4$ and $y(0)=0.3$.

$\eta_{2}=-0.0210<0$, and $\eta_{3}=-0.8543<0$. The conditions of Theorem 8 are satisfied, so the prey and predator are both extinct (see Figure 3).

Next we only change the density of the white noise to $\left(\sigma_{1}(1), \sigma_{2}(1)\right)=(0.3,0.2)$ and $\left(\sigma_{1}(2), \sigma_{2}(2)\right)=(0.5,0.4)$ and keep $\mu=0.15$. Simple calculation shows that $\eta_{4}=0.8622>0$, $\eta_{5}=0.9622>0$, and $\eta_{6}>0.6757>0$. The conditions of Theorem 10 are satisfied, so the prey and predator are persistent (see Figure 4).

It is easy to see from Figures 3 and 4 that the increase of the intensity of white noise can result in the extinction of prey and predator.
Case 2. Let $\mu=0.55,\left(\sigma_{1}(1), \sigma_{2}(1)\right)=(0.6,0.5)$, and $\left(\sigma_{1}(2), \sigma_{2}(2)\right)=(0.45,0.55)$, which gives $\eta_{1}=-2.4356<0$, $\eta_{2}=-0.3701<0$, and $\eta_{3}=-1.2035<0 . x(t)$ and $y(t)$ are extinct (see Figure 5).

Next we only change the amount of toxicant to $\mu=0.12$ and keep $\left(\sigma_{1}(1), \sigma_{2}(1)\right)=(0.6,0.5)$ and $\left(\sigma_{1}(2), \sigma_{2}(2)\right)=$ $(0.45,0.55)$, We note $\eta_{4}=1.0467>0, \eta_{5}=1.1467>0$, and $\eta_{6}>0.7255>0$. Thus $x(t)$ and $y(t)$ are persistent in mean (see Figure 6).

Figures 5 and 6 show that the increase of the amount of toxicant can also result in the extinction of the prey and predator. 

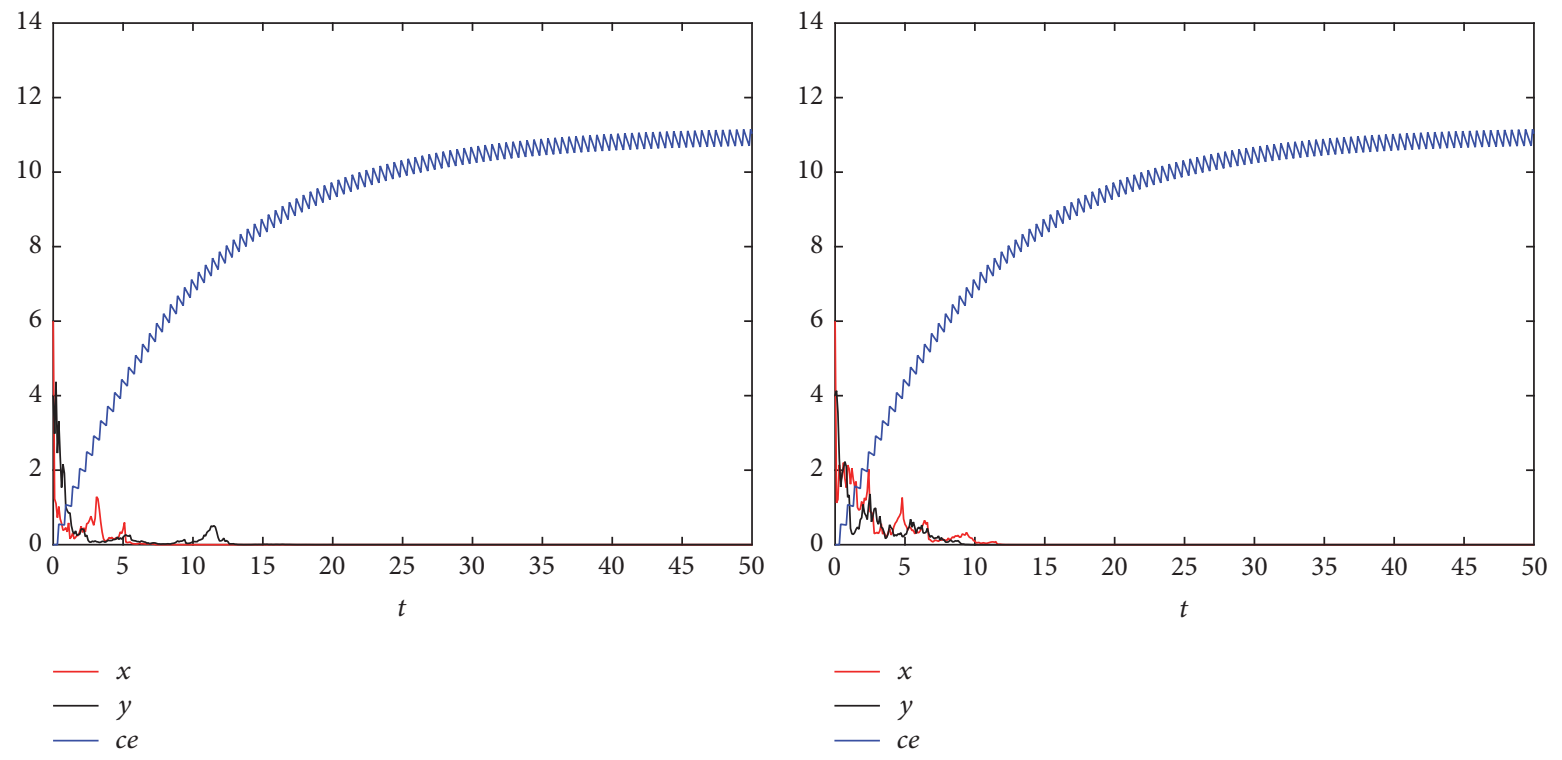

(a) $k=1$

(b) $k=2$

FIGURE 5: Sample paths of $x(t), y(t)$ with initial value $x(0)=6$ and $y(0)=4$.
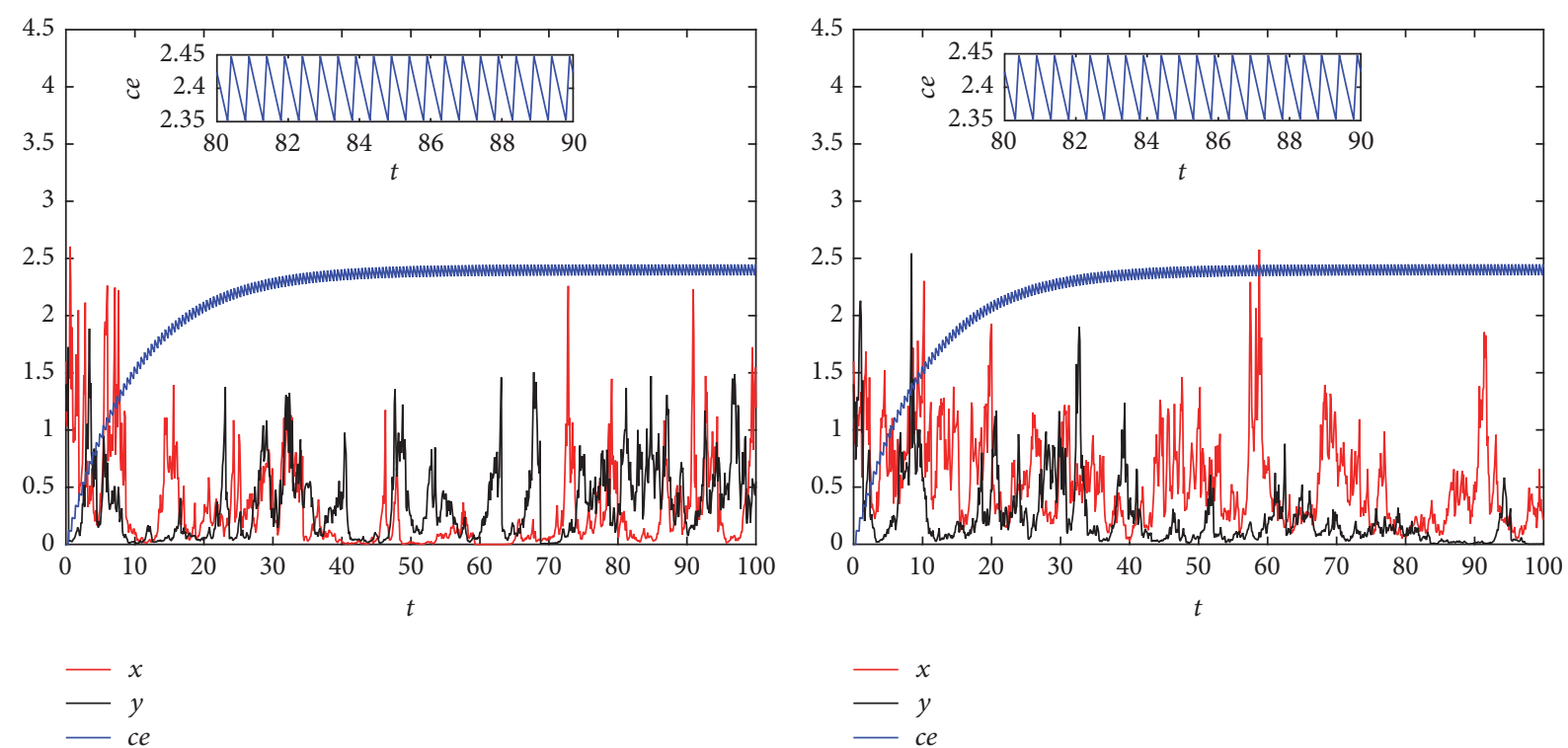
(a) $k=1$
(b) $k=2$

FIGURE 6: Sample paths of $x(t), y(t)$ with initial value $x(0)=1.6$ and $y(0)=1.4$.

\section{Conflicts of Interest}

The authors declare that there are no conflicts of interest regarding the publication of this paper.

\section{Acknowledgments}

This work is supported by Shandong Provincial Natural Science Foundation of China (no. ZR2015AQ001), the National Natural Science Foundation of China (no. 11371230), and
Research Funds for Joint Innovative Center for Safe and Effective Mining Technology and Equipment of Coal Resources by Shandong Province and SDUST (2014TDJH102).

\section{References}

[1] A. J. Lotka, "Contribution to the theory of periodic reactions," Journal of Physical Chemistry, vol. 14, no. 3, pp. 271-274, 1910.

[2] A. J. Lotka, "Analytical note on certain rhythmic relations in organic systems," Proceedings of the National Academy of Sciences, vol. 6, no. 7, pp. 410-415, 1920. 
[3] V. Volterra, "Variazioni e fluttuazioni del numero d'individui in specie animali conviventi," Rendiconti dell' Accademia dei Lincei, vol. 2, no. 1, pp. 31-113, 1926.

[4] J. Hofbauer and J. W.-H. So, "Multiple limit cycles for three dimensional Lotka-Volterra equations," Applied Mathematics Letters, vol. 7, no. 6, pp. 65-70, 1994.

[5] K. Gopalsamy, Stability and Oscillations in Delay Differential Equation of Population Dynamics, Mathematics and Its Applications, Springer Netherlands, Berlin, Germany, 1992.

[6] T. Zhang, W. Ma, X. Meng, and T. Zhang, "Periodic solution of a prey-predator model with nonlinear state feedback control," Applied Mathematics and Computation, vol. 266, pp. 95-107, 2015.

[7] Y. Kuang, Delay Differential Equations with Applications in Population Dynamics, Academic Press, Boston, Massachusetts, USA, 1993.

[8] T. Zhang, X. Meng, and T. Zhang, "Global analysis for a delayed siv model with direct and environmental transmissions," Journal of Applied Analysis and Computation, vol. 6, no. 2, pp. 479491, 2016.

[9] G. Huang, A. Liu, and U. Forys', "Global stability analysis of some nonlinear delay differential equations in population dynamics," Journal of Nonlinear Science, vol. 26, no. 1, pp. 2741, 2016.

[10] Z. Y. Lu and Y. Takeuchi, "Permanence and global attractivity for competitive Lotka-Volterra systems with delay," Nonlinear Analysis: Theory, Methods \& Applications, vol. 22, no. 7, pp. 847856, 1994.

[11] T. Zhang, T. Zhang, and X. Meng, "Stability analysis of a chemostat model with maintenance energy," Applied Mathematics Letters, vol. 68, pp. 1-7, 2017.

[12] A. Miao, J. Zhang, T. Zhang, and B. G. S. A. Pradeep, “Threshold dynamics of a stochastic SIR model with vertical transmission and vaccination," Computational and Mathematical Methods in Medicine, vol. 2017, pp. 1-10, 2017.

[13] T. Zhang, W. Ma, and X. Meng, "Impulsive control of a continuous-culture and flocculation harvest chemostat model," International Journal of Systems Science, pp. 1-11, 2017.

[14] X. Zhuo and F. Zhang, "Stability for a new discrete ratiodependent predator-prey system," Qualitative Theory of Dynamical Systems, pp. 1-14, 2017.

[15] C. S. Holling, "The components of predation as revealed by a study of small-mammal predation of the european pine sawfly," The Canadian Entomologist, vol. 91, no. 5, pp. 293-320, 1959.

[16] C. S. Holling, "Some characteristics of simple types of predation and parasitism," The Canadian Entomologist, vol. 91, no. 7, pp. 385-395, 1959.

[17] T. Zhang, J. Zhang, X. Meng, and T. Zhang, "Geometric analysis of a pest management model with holling's type iii functional response and nonlinear state feedback control," Nonlinear Dynamics, vol. 84, no. 3, pp. 1529-1539, 2016.

[18] J. R. Beddington, "Mutual interference between parasites or predators and its effect on searching efficiency," Journal of Animal Ecology, vol. 44, no. 2, pp. 331-340, 1975.

[19] D. L. DeAngelis, R. A. Goldstein, and R. V. O’Neill, “A model for tropic interaction," Ecology, vol. 56, no. 4, pp. 881-892, 1975.

[20] R. Arditi, N. Perrin, and H. Saah, "Functional response and heterogeneities: an experimental test with cladocerans," Oikos, vol. 60, no. 1, pp. 69-75, 1991.
[21] C. Jost and S. P. Ellner, "Testing for predator dependence in predator-prey dynamics: A non-parametric approach," Proceedings of the Royal Society B Biological Science, vol. 267, no. 1453, pp. 1611-1620, 2000.

[22] C. Jost and R. Arditi, "From pattern to process: Identifying predator-prey models from time-series data," Population Ecology, vol. 43, no. 3, pp. 229-243, 2001.

[23] G. T. Skalski and J. F. Gilliam, "Functional responses with predator interference: viable alternatives to the Holling type II model," Ecology, vol. 82, no. 11, pp. 3083-3092, 2001.

[24] Z. Qiu, J. Yu, and Y. Zou, "The asymptotic behavior of a chemostat model with the Beddington-DeAngelis functional response," Mathematical Biosciences, vol. 187, no. 2, pp. 175-187, 2004.

[25] R. S. Cantrell and C. Cosner, "On the dynamics of predator-prey models with the Beddington-DeAngelis functional response," Journal of Mathematical Analysis and Applications, vol. 257, no. 1, pp. 206-222, 2001.

[26] G. A. Polis and D. R. Strong, "Food web complexity and community dynamics," The American Naturalist, vol. 147, no. 5, pp. 813-846, 1996.

[27] K. McCann and A. Hastings, "Re-evaluating the omnivorystability relationship in food webs," Proceedings of the Royal Society B: Biological Sciences, vol. 264, no. 1385, pp. 1249-1254, 1997.

[28] T. Zhang, X. Meng, Y. Song, and T. Zhang, "A stage-structured predator-prey SI model with disease in the prey and impulsive effects," Mathematical Modelling and Analysis, vol. 18, no. 4, pp. 505-528, 2013.

[29] X. Li, X. Lin, and Y. Lin, "Lyapunov-type conditions and stochastic differential equations driven by g-brownian motion," Journal of Mathematical Analysis and Applications, vol. 439, no. 1, pp. 235-255, 2016.

[30] H.-J. Ma and T. Hou, "A separation theorem for stochastic singular linear quadratic control problem with partial information," Acta Mathematicae Applicatae Sinica, vol. 29, no. 2, pp. 303-314, 2013.

[31] Q. Liu, Y. Liu, and X. Pan, "Global stability of a stochastic predator-prey system with infinite delays," Applied Mathematics and Computation, vol. 235, pp. 1-7, 2014.

[32] T. Feng, X. Meng, L. Liu, and S. Gao, "Application of inequalities technique to dynamics analysis of a stochastic ecoepidemiology model," Journal of Inequalities and Applications, vol. 2016, no. 1, 327 pages, 2016.

[33] X. Meng, "Stability of a novel stochastic epidemic model with double epidemic hypothesis," Applied Mathematics and Computation, vol. 217, no. 2, pp. 506-515, 2010.

[34] X. Lin and R. Zhang, " $H_{\infty}$ control for stochastic systems with poisson jumps," Journal of Systems Science and Complexity, vol. 24, no. 4, pp. 683-700, 2011.

[35] A. Miao, X. Wang, T. Zhang, W. Wang, and B. Sampath Aruna Pradeep, "Dynamical analysis of a stochastic SIS epidemic model with nonlinear incidence rate and double epidemic hypothesis," Advances in Difference Equations, vol. 2017, pp. 127, 2017.

[36] J. Zhang, A. Miao, and T. Zhang, "Threshold dynamics of a stochastic chemostat model with two nutrients and one microorganism," Mathematical Problems in Engineering, vol. 2017, pp. 1-11, 2017.

[37] L. Liu and X. Meng, "Optimal harvesting control and dynamics of two-species stochastic model with delays," Advances in Difference Equations, vol. 2017, no. 1, pp. 1-17, 2017. 
[38] L. Yang and S. Zhong, "Global stability of a stage-structured predator-prey model with stochastic perturbation," Discrete Dynamics in Nature and Society, vol. 2014, Article ID 512817, pp. $1-8,2014$.

[39] Q. Liu, Q. Chen, and Y. Hu, "Analysis of a stochastic mutualism model," Communications in Nonlinear Science and Numerical Simulation, vol. 29, no. 1-3, pp. 188-197, 2015.

[40] L. Zu, D. Jiang, D. O’Regan, and B. Ge, "Periodic solution for a non-autonomous Lotka-Volterra predator-prey model with random perturbation," Journal of Mathematical Analysis and Applications, vol. 430, no. 1, pp. 428-437, 2015.

[41] D. Jiang, C. Ji, X. Li, and D. O’Regan, "Analysis of autonomous Lotka-Volterra competition systems with random perturbation," Journal of Mathematical Analysis and Applications, vol. 390, no. 2, pp. 582-595, 2012.

[42] R. Rudnicki and K. Pichór, "Influence of stochastic perturbation on prey-predator systems," Mathematical Biosciences, vol. 206, no. 1, pp. 108-119, 2007.

[43] M. Liu and K. Wang, "Persistence, extinction and global asymptotical stability of a non-autonomous predator-prey model with random perturbation," Applied Mathematical Modelling, vol. 36, no. 11, pp. 5344-5353, 2012.

[44] D. Jiang, N. Shi, and X. Li, "Global stability and stochastic permanence of a non-autonomous logistic equation with random perturbation," Journal of Mathematical Analysis and Applications, vol. 340, no. 1, pp. 588-597, 2008.

[45] X. Li and X. Mao, "Population dynamical behavior of nonautonomous Lotka-Volterra competitive system with random perturbation," Discrete and Continuous Dynamical Systems. Series A, vol. 24, no. 2, pp. 523-593, 2009.

[46] X. Lv, L. Wang, and X. Meng, "Global analysis of a new nonlinear stochastic differential competition system with impulsive effect," Advances in Difference Equations, vol. 2017, no. 1, pp. 1-19, 2017.

[47] G. Liu, X. Wang, X. Meng, and S. Gao, "Extinction and persistence in mean of a novel delay impulsive stochastic infected predator-prey system with jumps," Complexity, vol. 2017, pp. 1-17, 2017.

[48] X. Leng, T. Feng, and X. Meng, "Stochastic inequalities and applications to dynamics analysis of a novel SIVS epidemic model with jumps," Journal of Inequalities and Applications, vol. 2017, pp. 1-25, 2017.

[49] Q. Liu and Q. Chen, "Analysis of a stochastic delay predatorprey system with jumps in a polluted environment," Applied Mathematics and Computation, vol. 242, pp. 90-100, 2014.

[50] M. Liu and K. Wang, "Survival analysis of stochastic singlespecies population models in polluted environments," Ecological Modelling, vol. 220, no. 9-10, pp. 1347-1357, 2009.

[51] J. He and K. Wang, "The survival analysis for a population in a polluted environment," Nonlinear Analysis. Real World Applications. An International Multidisciplinary Journal, vol. 10, no. 3, pp. 1555-1571, 2009.

[52] Q. Han, D. Jiang, and C. Ji, "Analysis of a delayed stochastic predator-prey model in a polluted environment," Applied Mathematical Modelling, vol. 38, no. 13, pp. 3067-3080, 2014.

[53] W. Zhao, J. Li, T. Zhang, X. Meng, and T. Zhang, "Persistence and ergodicity of plant disease model with Markov conversion and impulsive toxicant input," Communications in Nonlinear Science and Numerical Simulation, vol. 48, pp. 70-84, 2017.

[54] X. Meng, L. Wang, and T. Zhang, "Global dynamics analysis of a nonlinear impulsive stochastic chemostat system in a polluted environment," Journal of Applied Analysis and Computation, vol. 6, no. 3, pp. 865-875, 2016.

[55] Z. Liu and Q. Liu, "Persistence and extinction of a stochastic delay predator-prey model in a polluted environment," Mathematica Slovaca, vol. 66, no. 1, pp. 95-106, 2016.

[56] B. Liu, L. Chen, and Y. Zhang, “The effects of impulsive toxicant input on a population in a polluted environment," Journal of Biological Systems, vol. 11, no. 3, pp. 265-274, 2003.

[57] D. Jiang, Q. Zhang, T. Hayat, and A. Alsaedi, "Periodic solution for a stochastic non-autonomous competitive LotkaVolterra model in a polluted environment," Physica A. Statistical Mechanics and its Applications, vol. 471, pp. 276-287, 2017.

[58] M. Liu and K. Wang, "Persistence and extinction of a stochastic single-specie model under regime switching in a polluted environment," Journal of Theoretical Biology, vol. 264, no. 3, pp. 934-944, 2010.

[59] H. Liu and Z. Ma, "The threshold of survival for system of two species in a polluted environment," Journal of Mathematical Biology, vol. 30, no. 1, pp. 49-61, 1991.

[60] X. Zhang, D. Jiang, A. Alsaedi, and T. Hayat, "Stationary distribution of stochastic SIS epidemic model with vaccination under regime switching," Applied Mathematics Letters, vol. 59, pp. 87-93, 2016.

[61] T. G. Hallam, C. E. Clark, and R. R. Lassiter, "Effects of toxicants on populations: a qualitative approach I. Equilibrium environmental exposure," Ecological Modelling, vol. 18, no. 3-4, pp. 291-304, 1983.

[62] T. G. Hallam, C. E. Clark, and G. S. Jordan, "Effects of toxicants on populations: A qualitative approach II. first order kinetics," Journal of Mathematical Biology, vol. 18, no. 1, pp. 25-37, 1983.

[63] T. G. Hallam and J. T. de Luna, "Effects of toxicants on populations: A qualitative. Approach III. Environmental and food chain pathways," Journal of Theoretical Biology, vol. 109, no. 3, pp. 411-429, 1984.

[64] Z. E. Ma, G. R. Cui, and W. D. Wang, "Persistence and extinction of a population in a polluted environment," Mathematical Biosciences, vol. 101, no. 1, pp. 75-97, 1990.

[65] Z. Zhao and X. Wu, "Nonlinear analysis of a delayed stagestructured predator-prey model with impulsive effect and environment pollution," Applied Mathematics and Computation, vol. 232, Supplement C, pp. 1262-1268, 2014.

[66] X. Meng, R. Liu, and T. Zhang, "Adaptive dynamics for a nonautonomous Lotka-Volterra model with size-selective disturbance," Nonlinear Analysis. Real World Applications, vol. 16, pp. 202-213, 2014.

[67] S. B. Hsu and P. Waltman, "Competition in the chemostat when one competitor produces a toxin," Japan Journal of Industrial and Applied Mathematics, vol. 15, no. 3, pp. 471-490, 1998.

[68] B. Liu, S. Luan, and Y. Gao, "Modeling the dynamics of a single-species model with pollution treatment in a polluted environment," Discrete Dynamics in Nature and Society, vol. 2013, Article ID 412409, pp. 107-115, 2013.

[69] Y. Lenbury and N. Tumrasvin, "Singular perturbation analysis of a model for the effect of toxicants in single-species systems," Mathematical and Computer Modelling, vol. 31, no. 4-5, pp. 125$134,2000$.

[70] Q. Luo and X. Mao, "Stochastic population dynamics under regime switching II," Journal of Mathematical Analysis and Applications, vol. 355, no. 2, pp. 577-593, 2009.

[71] Y. Zhao, S. Yuan, and T. Zhang, "The stationary distribution and ergodicity of a stochastic phytoplankton allelopathy model 
under regime switching," Communications in Nonlinear Science and Numerical Simulation, vol. 37, pp. 131-142, 2016.

[72] X. Li, A. Gray, D. Jiang, and X. Mao, "Sufficient and necessary conditions of stochastic permanence and extinction for stochastic logistic populations under regime switching," Journal of Mathematical Analysis and Applications, vol. 376, no. 1, pp. 1128, 2011.

[73] A. Lahrouz, A. Settati, and P. S. Mandal, "Dynamics of a switching diffusion modified Leslie-Gower predator-prey system with Beddington-DeAngelis functional response," Nonlinear Dynamics, vol. 85, no. 2, pp. 853-870, 2016.

[74] M. Liu and K. Wang, "Asymptotic properties and simulations of a stochastic logistic model under regime switching," Mathematical and Computer Modelling, vol. 54, no. 9-10, pp. 2139-2154, 2011.

[75] M. Liu and K. Wang, "The threshold between permanence and extinction for a stochastic Logistic model with regime switching," Applied Mathematics and Computation, vol. 43, no. 1-2, pp. 329-349, 2013.

[76] H. Liu, X. Li, and Q. Yang, "The ergodic property and positive recurrence of a multi-group Lotka-Volterra mutualistic system with regime switching," Systems \& Control Letters, vol. 62, no. 10, pp. 805-810, 2013.

[77] A. Settati and A. Lahrouz, "Stationary distribution of stochastic population systems under regime switching," Applied Mathematics and Computation, vol. 244, pp. 235-243, 2014.

[78] R. Z. Khasminskii, C. Zhu, and G. Yin, "Stability of regimeswitching diffusions," Stochastic Processes and Their Applications, vol. 117, no. 8, pp. 1037-1051, 2007.

[79] X. Li, D. Jiang, and X. Mao, "Population dynamical behavior of Lotka-Volterra system under regime switching," Journal of Computational and Applied Mathematics, vol. 232, no. 2, pp. 427-448, 2009.

[80] M. Liu and K. Wang, "Asymptotic properties and simulations of a stochastic logistic model under regime switching," Mathematical and Computer Modelling, vol. 55, no. 3-4, pp. 405-418, 2012.

[81] Y. Zhao and W. Zhang, "Observer-based controller design for singular stochastic markov jump systems with state dependent noise," Journal of Systems Science and Complexity, vol. 29, no. 4, pp. 946-958, 2016.

[82] H. Ma and Y. Jia, "Stability analysis for stochastic differential equations with infinite Markovian switchings," Journal of Mathematical Analysis and Applications, vol. 435, no. 1, pp. 593-605, 2016.

[83] T. Zhang, W. Ma, and X. Meng, "Global dynamics of a delayed chemostat model with harvest by impulsive flocculant input," Advances in Difference Equations, vol. 2017, pp. 1-17, 2017.

[84] X. Mao, Stochastic Differential Equations and Applications, Horwood, Chichester, UK, 2nd edition, 1997.

[85] D. Li and D. Xu, "Periodic solutions of stochastic delay differential equations and applications to logistic equation and neural networks," Journal of the Korean Mathematical Society, vol. 50, no. 6, pp. 1165-1181, 2013. 


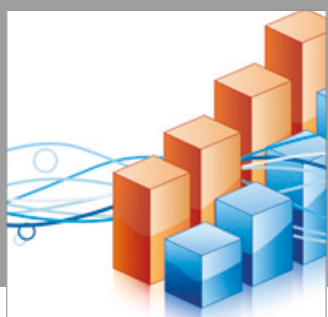

Advances in

Operations Research

vatersals

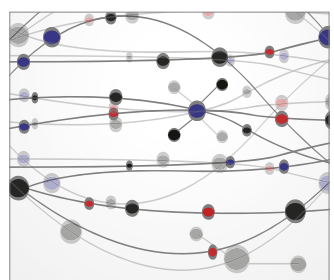

\section{The Scientific} World Journal
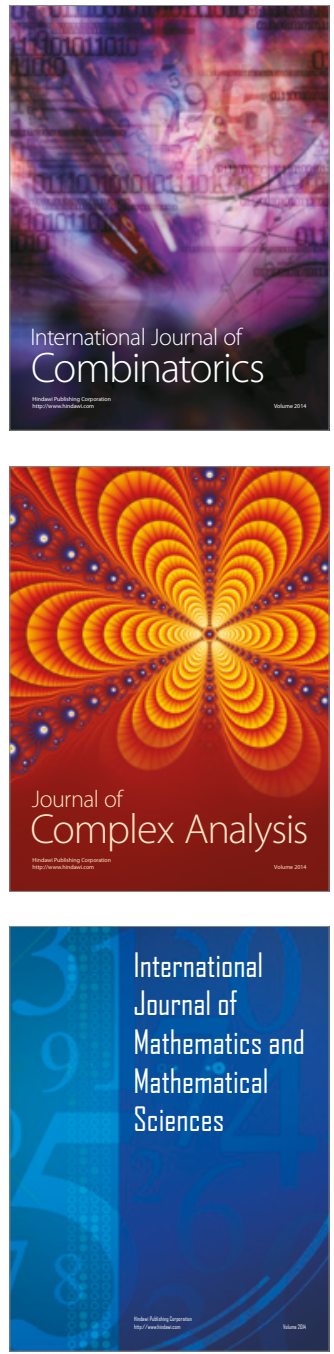
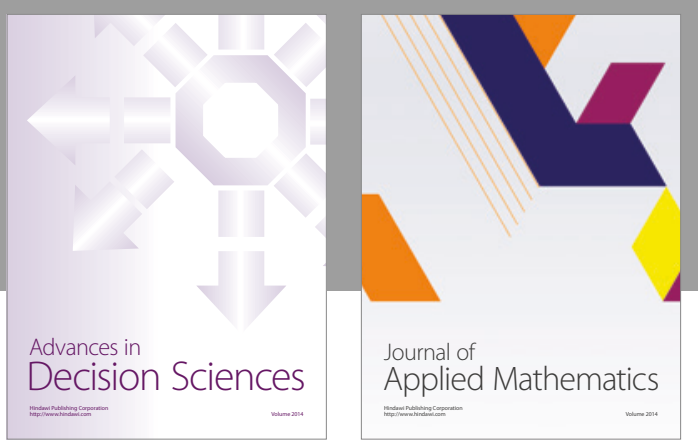

Algebra

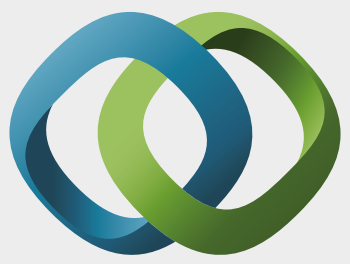

\section{Hindawi}

Submit your manuscripts at

https://www.hindawi.com
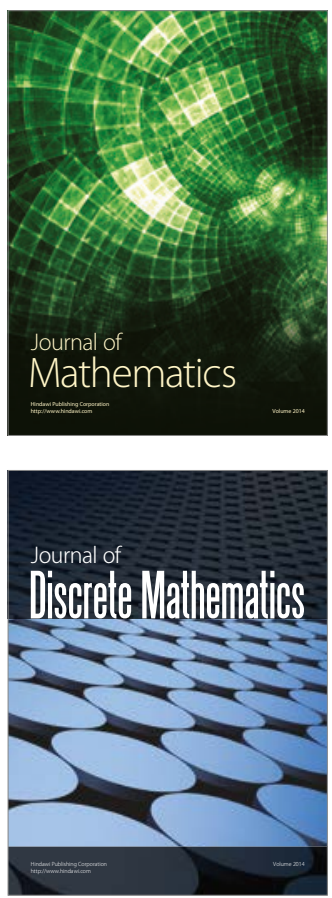

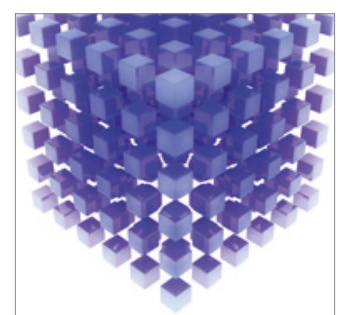

Mathematical Problems in Engineering
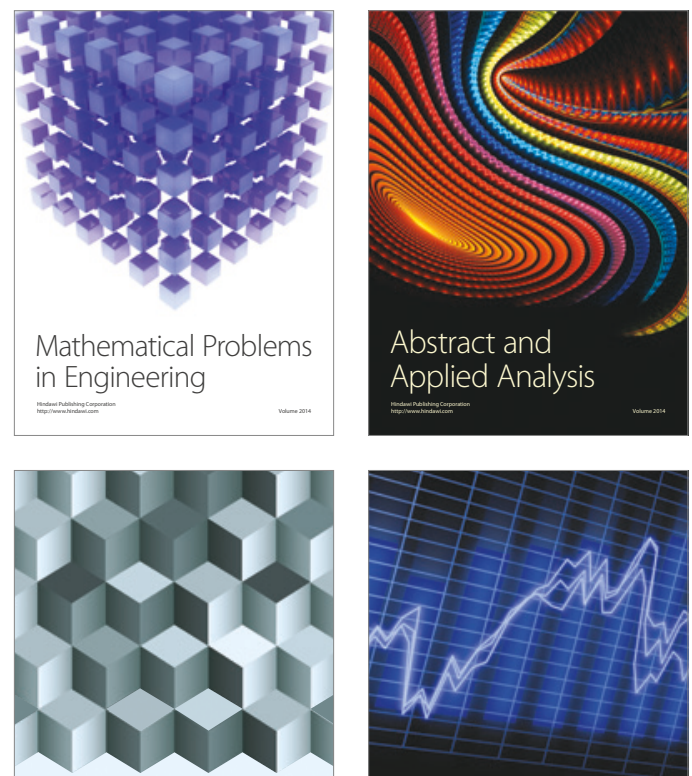

Journal of

Function Spaces

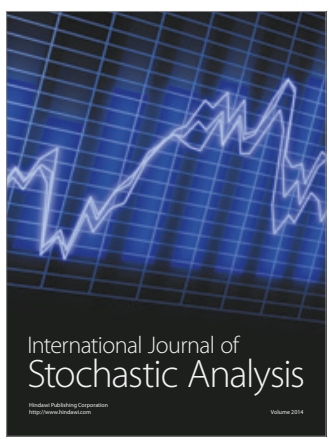

Probability and Statistics
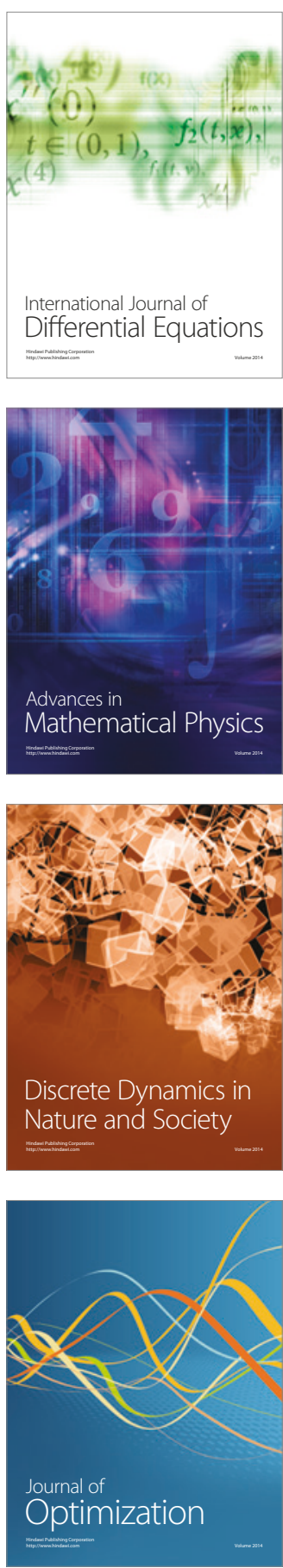